Cochrane Database of Systematic Reviews

\title{
Hearing aids for mild to moderate hearing loss in adults (Review)
}

Ferguson MA, Kitterick PT, Chong LY, Edmondson-Jones M, Barker F, Hoare DJ

Ferguson MA, Kitterick PT, Chong LY, Edmondson-Jones M, Barker F, Hoare DJ.

Hearing aids for mild to moderate hearing loss in adults.

Cochrane Database of Systematic Reviews 2017, Issue 9. Art. No.: CD012023.

DOI: 10.1002/14651858.CD012023.pub2.

www.cochranelibrary.com 
TABLE OF CONTENTS

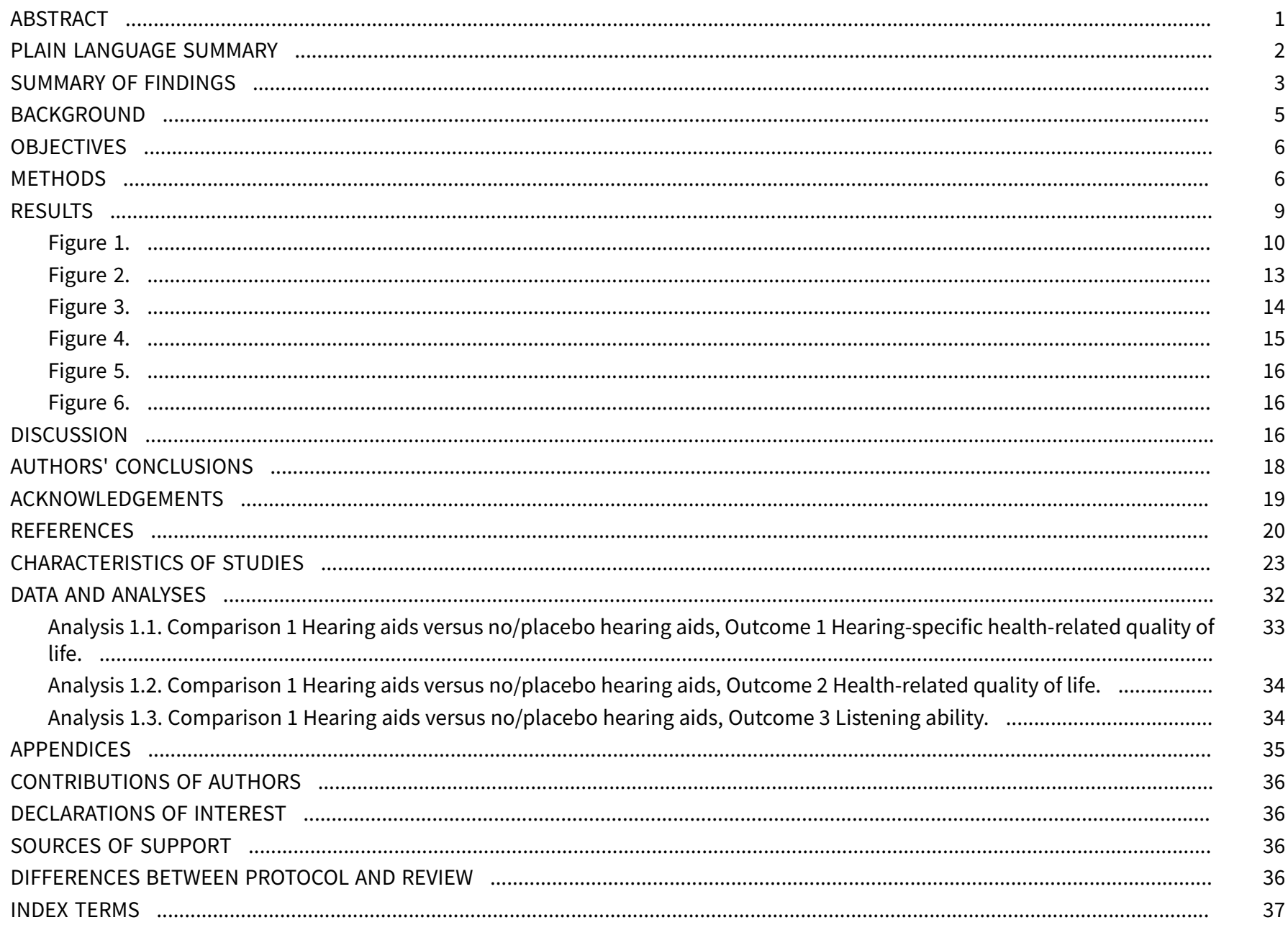


[Intervention Review]

\section{Hearing aids for mild to moderate hearing loss in adults}

Melanie A Ferguson 1,2, Pádraig T Kitterick², Lee Yee Chong³, Mark Edmondson-Jones², Fiona Barker4, Derek J Hoare²

1NIHR Nottingham Biomedical Research Centre, Nottingham University Hospitals NHS Trust, Nottingham, UK. ${ }^{2}$ NIHR Nottingham Biomedical Research Centre, Division of Clinical Neuroscience, School of Medicine, University of Nottingham, Nottingham, UK. ${ }^{3}$ UK Cochrane Centre, Oxford, UK. ${ }^{4}$ Department of Clinical and Experimental Medicine, University of Surrey, Guildford, UK

Contact: Melanie A Ferguson, NIHR Nottingham Biomedical Research Centre, Nottingham University Hospitals NHS Trust, Ropewalk House, 113 The Ropewalk, Nottingham, NG15DU, UK. melanie.ferguson@nottingham.ac.uk.

Editorial group: Cochrane ENT Group.

Publication status and date: New, published in Issue 9, 2017.

Citation: Ferguson MA, Kitterick PT, Chong LY, Edmondson-Jones M, Barker F, Hoare DJ. Hearing aids for mild to moderate hearing loss in adults. Cochrane Database of Systematic Reviews 2017, Issue 9. Art. No.: CD012023. DOI: 10.1002/14651858.CD012023.pub2.

Copyright @ 2017 The Cochrane Collaboration. Published by John Wiley \& Sons, Ltd.

\section{A B S T R A C T}

\section{Background}

The main clinical intervention for mild to moderate hearing loss is the provision of hearing aids. These are routinely offered and fitted to those who seek help for hearing difficulties. By amplifying and improving access to sounds, and speech sounds in particular, the aim of hearing aid use is to reduce the negative consequences of hearing loss and improve participation in everyday life.

\section{Objectives}

To evaluate the effects of hearing aids for mild to moderate hearing loss in adults.

\section{Search methods}

The Cochrane ENT Information Specialist searched the ENT Trials Register; the Cochrane Register of Studies Online; MEDLINE; PubMed; EMBASE; CINAHL; Web of Science; ClinicalTrials.gov; ICTRP and additional sources for published and unpublished trials. The date of the search was 23 March 2017.

\section{Selection criteria}

Randomised controlled trials (RCTs) of hearing aids compared to a passive or active control in adults with mild to moderate hearing loss.

\section{Data collection and analysis}

We used the standard methodological procedures expected by Cochrane. The primary outcomes in this review were hearing-specific health-related quality of life and the adverse effect pain. Secondary outcomes were health-related quality of life, listening ability and the adverse effect noise-induced hearing loss. We used GRADE to assess the quality of the evidence for each outcome; this is indicated in italics.

\section{Main results}

We included five RCTs involving 825 participants. The studies were carried out in the USA and Europe, and were published between 1987 and 2017. Risk of bias across the studies varied. Most had low risk for selection, reporting and attrition bias, and a high risk for performance and detection bias because blinding was inadequate or absent.

All participants had mild to moderate hearing loss. The average age across all five studies was between 69 and 83 years. The duration of the studies ranged between six weeks and six months. 
There was a large beneficial effect of hearing aids on hearing-specific health-related quality of life associated with participation in daily life as measured using the Hearing Handicap Inventory for the Elderly (HHIE, scale range 1 to 100) compared to the unaided/placebo condition (mean difference (MD) -26.47, 95\% confidence interval (CI) -42.16 to $-10.77 ; 722$ participants; three studies) (moderate-quality evidence).

There was a small beneficial effect of hearing aids on general health-related quality of life (standardised mean difference (SMD) - $0.38,95 \%$ $\mathrm{Cl}-0.55$ to $-0.21 ; 568$ participants; two studies) (moderate-quality evidence). There was a large beneficial effect of hearing aids on listening ability (SMD -1.88, 95\% Cl -3.24 to -0.52; 534 participants; two studies) (moderate-quality evidence).

Adverse effects were measured in only one study (48 participants) and none were reported (very low-quality evidence).

\section{Authors' conclusions}

The available evidence concurs that hearing aids are effective at improving hearing-specific health-related quality of life, general healthrelated quality of life and listening ability in adults with mild to moderate hearing loss. The evidence is compatible with the widespread provision of hearing aids as the first-line clinical management in those who seek help for hearing difficulties. Greater consistency is needed in the choice of outcome measures used to assess benefits from hearing aids. Further placebo-controlled studies would increase our confidence in the estimates of these effects and ascertain whether they vary according to age, gender, degree of hearing loss and type of hearing aid.

\section{PLAIN LANGUAGE SUMMARY}

\section{Hearing aids for mild to moderate hearing loss in adults}

\section{Review question}

We reviewed the evidence on the effects that hearing aids have on everyday life in adults with mild to moderate hearing loss. We were interested in (1) a person's ability to take part in everyday situations, (2) general health-related quality of life, (3) ability to listen to other people, and (4) harm, such as pain or over-exposure to noise.

\section{Background}

Hearing loss is very common and adults with hearing loss may be offered hearing aids. These devices increase the loudness, and may improve the clarity, of sounds so that they are easier to hear. The main goal of hearing aids is to reduce the impact of hearing loss and to improve a person's ability to take part in everyday life. Although hearing aids are the most common technology for adults with hearing loss and are in widespread use, it is not clear how beneficial they are.

\section{Study characteristics}

The evidence is up to date to 23 March 2017. We found five clinical studies involving 825 adults with mild to moderate hearing loss who were randomly given either hearing aids, no hearing aids or placebo hearing aids. Studies involved older adults with the average age within studies between 69 and 83 years. The duration of the studies was between six weeks and six months.

\section{Key results}

We found evidence in three studies that hearing aids have a large beneficial effect in improving the ability of adults with mild to moderate hearing loss to take part in everyday situations. Hearing aids have a small beneficial effect in improving general health-related quality of life, such as physical, social, emotional and mental well-being, and have a large effect in improving the ability to listen to other people.

Only one study attempted to measure harms due to hearing aids. None were reported.

\section{Quality of the evidence}

We judged the evidence that hearing aids improve the ability to take part in everyday situations, improve general health-related quality of life and improve listening ability to be of moderate quality. This means that while we are reasonably confident that the reported benefits of hearing aids are real, there is a possibility that if further studies are conducted the size of the benefit might differ. We judged the quality of evidence for harms to be very low, because this was only measured in one small study.

\section{Conclusions}

We found that hearing aids improve the ability of adults with mild to moderate hearing loss to take part in everyday life, their general quality of life and their ability to listen to other people. If an adult with mild to moderate hearing loss seeks help for their hearing difficulties, hearing aids are an effective clinical option. It is important that future studies measure benefits consistently and report benefits separately for different age groups, genders, levels of hearing loss and types of hearing aids. 
SUMMARY OF FINDINGS

Summary of findings for the main comparison. Hearing aids versus no hearing aids for mild to moderate hearing loss in adults

Hearing aids versus no hearing aids for mild to moderate hearing loss in adults

Patient or population: adults with mild to moderate hearing loss

Setting: audiology services and clinics

Intervention: hearing aids

Comparison: no hearing aids (waiting list) or placebo hearing aids

\begin{tabular}{|c|c|c|c|c|c|}
\hline \multirow{2}{*}{$\begin{array}{l}\text { Outcomes } \\
\text { No. of participants } \\
\text { (studies) }\end{array}$} & \multicolumn{3}{|c|}{ Anticipated absolute effects $(95 \% \mathrm{Cl})$} & \multirow[t]{2}{*}{ Quality } & \multirow[t]{2}{*}{ What happens* } \\
\hline & $\begin{array}{l}\text { Without hearing } \\
\text { aids }\end{array}$ & With hearing aids & Difference & & \\
\hline $\begin{array}{l}\text { Hearing-specific HRQoL } \\
\text { assessed with: HHIE (range } 0 \text { to } \\
\text { 100) } \\
\text { Follow-up: range } 6 \text { to } 16 \text { weeks } \\
\text { No. of participants: } 722 \text { (3 RCTs) }\end{array}$ & $\begin{array}{l}\text { The mean hear- } \\
\text { ing-specific HRQoL } \\
\text { score was } 39\end{array}$ & - & $\begin{array}{l}\text { Mean } 26 \text { lower ( } 42 \\
\text { to } 11 \text { lower) }\end{array}$ & $\begin{array}{l}\oplus \oplus \oplus \ominus \\
\text { MODERATE } 1,2,3,4\end{array}$ & $\begin{array}{l}\text { Lower score indicates better hearing-specif- } \\
\text { ic HRQoL. The mean difference corresponds } \\
\text { to a large effect size (SMD }-1.38,95 \% \mathrm{Cl}-2.02 \\
\text { to }-0.75 \text { ) favouring hearing aids. }\end{array}$ \\
\hline $\begin{array}{l}\text { Health-related QoL } \\
\text { assessed with: WHO-DAS II (range } \\
0 \text { to } 100 \text { ) and the SELF (range } 54 \\
\text { to } 216 \text { ) } \\
\text { Follow-up: range } 2 \text { months to } 16 \\
\text { weeks } \\
\text { No. of participants: } 568 \text { ( } 2 \text { RCTs) }\end{array}$ & - & - & $\begin{array}{l}\text { SMD } 0.38 \text { lower } \\
\text { (0.55 lower to } 0.21 \\
\text { lower) }\end{array}$ & $\begin{array}{l}\oplus \oplus \oplus \ominus \\
\text { MODERATE } 1,4\end{array}$ & $\begin{array}{l}\text { Lower score indicates better HRQoL. The } \\
\text { SMD corresponds to a small effect size } \\
\text { favouring hearing aids, which is equiva- } \\
\text { lent to a } 6 \text {-point decrease (9- to 3-point de- } \\
\text { crease) on the } 0 \text { to } 100 \text { scale of the WHO- } \\
\text { DAS II5. }\end{array}$ \\
\hline $\begin{array}{l}\text { Listening ability } \\
\text { assessed with: PHAP (range } 0 \text { to } \\
\text { 1) and APHAB (range } 0 \text { to } 100 \text { ) } \\
\text { Follow-up: } 6 \text { weeks to } 2 \text { months } \\
\text { No. of participants: } 534 \text { ( } 2 \text { RCTs) }\end{array}$ & - & - & $\begin{array}{l}\text { SMD } 1.88 \text { lower } \\
\text { (3.24 lower to } 0.52 \\
\text { lower) }\end{array}$ & $\begin{array}{l}\oplus \oplus \oplus \ominus \\
\text { MODERATE } 1,2,3,4\end{array}$ & $\begin{array}{l}\text { Lower score indicates improved listening } \\
\text { ability. The SMD corresponds to a large ef- } \\
\text { fect size favouring hearing aids, which is } \\
\text { equivalent to a } 29 \text {-point decrease ( } 50 \text { - to } 8 \text { - } \\
\text { point decrease) on the } 0 \text { to } 100 \text { scale of the } \\
\text { APHAB6. }\end{array}$ \\
\hline Adverse effect - pain & $\begin{array}{l}\text { Adverse effects relat } \\
\text { were reported. }\end{array}$ & to pain were meas & din 1 study: none & $\oplus \ominus \ominus \ominus$ & $\begin{array}{l}\text { There was too little information to estimate } \\
\text { the risk of pain. }\end{array}$ \\
\hline
\end{tabular}


Adverse effect - noise-induced

hearing loss

No. of participants: 48

(1 RCT)

${ }^{*}$ The equivalent change in the intervention group (and its 95\% confidence interval) is based on the standard deviation in the comparison group from a representative study (see footnotes for each outcome) and the relative effect of the intervention (and its $95 \% \mathrm{Cl}$ ).

APHAB: Abbreviated Profile of Hearing Aid Benefit; CI: confidence interval; HHIE: Hearing Handicap Inventory for the Elderly; HRQoL: health-related quality of life; MD: mean difference; PHAP: Profile of Hearing Aid Performance; RCT: randomised controlled trial; SELF: Self Evaluation of Life Function; SMD: standardised mean difference; WHO-DAS II: WHO Disability Assessment Schedule II

\section{GRADE Working Group grades of evidence}

High quality: We are very confident that the true effect lies close to that of the estimate of the effect

Moderate quality: We are moderately confident in the effect estimate: The true effect is likely to be close to the estimate of the effect, but there is a possibility that it is substantially different

Low quality: Our confidence in the effect estimate is limited: The true effect may be substantially different from the estimate of the effect

Very low quality: We have very little confidence in the effect estimate: The true effect is likely to be substantially different from the estimate of effect

${ }^{1}$ Quality of evidence downgraded by one level because all studies have either a rating of unclear and/or high risk bias in at least one of these domains: selection bias, performance and/or detection bias.

2We considered downgrading for inconsistency due to observed statistical heterogeneity but we did not apply this. The data consistently showed large beneficial effects of using hearing aids for mild to moderate hearing loss despite the apparent differences in study designs and populations. Our confidence in the size of the effect is not affected.

3 We considered downgrading due to indirectness as some data were obtained after a short follow-up period (six weeks) but we did not apply this. Large beneficial effects were observed regardless of duration of follow-up.

${ }^{4}$ We considered downgrading due to indirectness as some analyses included data from male military veterans but we did not apply this. Effect sizes were consistent within each outcome despite differences in study samples and designs (small beneficial effect for HRQoL; large beneficial effect for hearing-specific HRQoL and listening ability).

5Equivalent change calculated assuming a standard deviation of 15.99 in WHO-DAS II scores in the no hearing aid group.

6 Equivalent change calculated assuming a standard deviation of 15.30 in APHAB scores in the no hearing aid group.

7 Very serious imprecision as the sample size was very small. There was serious indirectness because only people with mild to moderate Alzheimer's disease were included in the study. 


\section{B A C K G R O U N D}

\section{Description of the condition}

Hearing loss is the most prevalent sensory deficit (Mathers 2000), and represents a major public health issue with substantial economic and societal costs. Untreated, adult hearing loss results in communication difficulties that can lead to social isolation and withdrawal, depression and reduced quality of life (Davis 2007). Hearing loss is also associated with an increased risk of dementia, although currently the underlying mechanism is unknown (Lin 2011).

According to the World Health Organization, hearing loss is the 13th most common global disease burden and the third leading cause of years lived with disability (YLD) (WHO 2008). Disabling hearing loss is estimated to affect 360 million people globally (5.3\% of the world's population) (WHO 2012a). The prevalence of hearing loss increases with age (Akeroyd 2014), and given the ageing society it is predicted that by 2030 adult-onset hearing loss will be the seventh largest disease burden, above diabetes and human immunodeficiency virus (HIV) (WHO 2008).

Epidemiological data suggest that the majority of cases of hearing loss in adults are sensorineural in nature (92\%) and occur bilaterally (94.8\%) (Cruickshanks 1998). There are numerous definitions of hearing loss across different countries and organisations (Timmer 2015). In this review, hearing loss is defined according to averaged pure-tone thresholds across combinations of frequencies $(0.5 \mathrm{kHz}$, $1.0 \mathrm{kHz}, 2.0 \mathrm{kHz}$ and $4.0 \mathrm{kHz}$ ), and grades of hearing impairment are consistent with the World Health Organization (Mathers 2000). The majority of hearing losses (92\%) are those that are defined as mild or moderate (AoHL 2015). Mild (or slight) hearing loss is indicated as 26 to $40 \mathrm{~dB}$ hearing level $(\mathrm{HL})$ and described as the ability to hear and repeat words spoken in a normal voice at one metre. Moderate hearing loss is indicated as 41 to $60 \mathrm{~dB} \mathrm{HL}$ and described as the ability to hear and repeat words using a raised voice at one metre (Mathers 2000). In addition to a loss of hearing sensitivity there may be additional sensory deficits of temporal and spectral processing that contribute to speech perception (Hopkins 2011), not necessarily captured by pure-tone audiometry.

\section{Description of the intervention}

There are no effective medical or surgical treatments for mild to moderate sensorineural hearing loss (Chisolm 2007), so the main clinical intervention is the use of acoustic hearing aids (Kochkin 2009). It was estimated that in 2012, 11 million hearing aids were sold worldwide (Kirkwood 2013). Hearing aids detect and amplify sound, and deliver an amplified acoustic signal via air conduction to the external auditory canal on the same side as the signals are detected. Hearing aids are described according to where they are worn (e.g. behind-the-ear, in-the-ear, in-the-canal, completely-inthe-canal) or classified by their technology (i.e. analogue, digitally programmable analogue or digital hearing aids) (Dillon 2012). Hearing aids are typically fitted by healthcare professionals who have been trained in audiology or the dispensing of hearing aids.

Hearing aid fittings can be unilateral or bilateral and they are typically programmed according to the user's pure-tone hearing thresholds using hearing aid prescription formulae (Mueller 2005). Changes to the hearing aid programme may be made according to subjective preference for sound quality, such as the perceived loudness of sounds or the clarity of speech (McArdle 2005). Hearing aid orientation that includes information on hearing aid use and care, expectations and limitations is typically offered as usual care (Boothroyd 2007; Reese 2005).

The use of hearing aids to amplify sounds does not necessarily restore hearing function. Frequency response characteristics of hearing aids, distortions arising from peak clipping, poor clarity or loudness of speech can all have an impact on successful listening (Dillon 2012). As hearing aids amplify all sounds, not just speech sounds, their use can lead to continued communication difficulties, particularly in noisy backgrounds (Picou 2013). These and other reasons can lead to non-use of hearing aids (McCormack 2013), with estimates of non-use varying from $3 \%$ (Bertoli 2009) to $24 \%$ (Lupsakko 2005). Recent studies have shown non-use at $10 \%$ (Aazh 2015) and 15.5\% (Solheim 2017), consistent with commercial surveys (4.7\% to 7\% (Hougaard 2011), 12.4\% (Kochkin 2010)). Additional interventions may be used to promote the use of hearing aids in people with hearing loss (Barker 2016).

\section{How the intervention might work}

The primary function of hearing aids is to amplify and improve the audibility of sounds, and speech in particular. However, improving the audibility of sounds or speech signals forms only one element within the broader concept of rehabilitating a person with hearing loss, where the overall aim is to reduce the negative consequences of hearing loss and improve communication. In order to communicate effectively, an individual needs to access the acoustic information (hearing, a passive process), employ attention and intention (listening, an active process), correctly interpret the acoustic and linguistic information (comprehension, a unidirectional process) and use and transmit this information effectively (communication, a bidirectional process) (Kiessling 2003). These processes can be mapped onto the International Classification of Functioning, Disability and Health (ICF, WHO 2001), which provides a theoretical framework upon which to measure the success of amplification using hearing aids.

Based on the ICF Core Set (Danermark 2013), the goal of amplification with hearing aids where there is mild to moderate hearing loss is to reduce the auditory deficits associated with body functions and structures, thereby reducing activity limitations and participation restrictions (Chisolm 2007; Kiessling 2003). Participation has been defined as involvement in a life situation, especially engagement in a social domain, such as family relationships, community life, employment, education and recreation and leisure (Danermark 2013; Resnik 2009; WHO 2002). Hearing-related participation also has an emotional component, particularly feelings of social isolation and loneliness (Ventry 1982).

Improvements in the ability of a patient with hearing aids to detect and discriminate sounds and speech can be measured by acoustic outcomes (e.g. free-field threshold and speech audiometry). The consequences of these improvements in terms of activities and participation can then be measured by patient-reported outcomes such as self-report questionnaires, which can be defined as either disease-specific (e.g. hearing) or generic (e.g. health-related quality of life). Generic health-related quality of life measures generally show limited benefit from hearing aids as they lack sensitivity to the consequences of hearing loss (Joore 2002; Joore 2003; Stark 2004). There is, however, some evidence that the Health Utilities Index Mark 3 (HUI3) is useful (Barton 2004; Davis 2007). Currently, 
there is a lack of consensus on the optimal set of outcome measures to use in hearing research (Granberg 2014).

\section{Why it is important to do this review}

Hearing aids are routinely offered and fitted for people with hearing loss. It might seem obvious that such an intervention is bound to be associated with an improvement in a patient's ability to hear and to communicate, but is this true? If there is an improvement in a patient's ability to hear and communicate, how big an improvement is it? There is little high-level evidence to answer these questions and to inform discussions around the effectiveness of hearing aids, their provision within a population and the approach to be taken by those who might fund such provision.

There are no recent or ongoing systematic reviews that provide the high-level evidence to inform clinical decision-making on this important topic. A previous systematic review of the published evidence included randomised controlled trials (RCTs) and nonrandomised trials published up to August 2004 that met specific criteria. It sought to address a specific objective: to determine if the use of hearing aids compared to the non-use of hearing aids resulted in improvements in health-related quality of life for adults with sensorineural hearing loss using disease-specific and generic instruments (Chisolm 2007). The authors reported that there were only two RCTs suitable for inclusion at that time, although only one trial randomised the whole patient sample (Mulrow 1990), therefore limiting the generalisability of the findings and the robustness of the conclusions. A more recent systematic review that investigated hearing aid benefit in those with mild sensorineural hearing loss included 10 studies, but none of these were RCTs (Johnson 2016).

The present review does not compare the evidence for the bilateral versus unilateral fitting of hearing aids, for which there is an ongoing Cochrane Review (Browning 2017).

\section{O B JE C T IVES}

To evaluate the effects of hearing aids for mild to moderate hearing loss in adults.

\section{METHODS}

\section{Criteria for considering studies for this review}

\section{Types of studies}

We included randomised controlled trials and used the first treatment period of cross-over trials (i.e. treated as a parallel-arm trial) provided the study reporting permitted, where the unit of analysis was the individual participant.

\section{Types of participants}

Adults ( $\geq 18$ years old) who have mild to moderate hearing loss. For inclusion, hearing loss of participants had to be either described qualitatively as 'mild' and/or 'moderate', or the study group had to be quantitatively defined as having an average pure-tone threshold within the mild or moderate ranges of hearing loss as defined by the WHO (mild: 26 to $40 \mathrm{~dB}$ HL inclusive; moderate: 41 to $60 \mathrm{~dB} \mathrm{HL}$ inclusive). Here, an average pure-tone threshold is defined as the mean of at least two octave frequencies up to and including $4 \mathrm{kHz}$.

\section{Types of interventions}

Acoustic hearing aids, irrespective of where they are worn or the type of technology (analogue or digital).

We excluded hearing aids or implantable devices whose primary purpose is to deliver bone conduction sound or those that detect and deliver sound via air conduction to the contralateral ear.

The comparisons of interest were hearing aids versus either a passive control (no intervention, waiting list control; these were pooled in the meta-analysis) or an active control that involved:

- information/education only, listening tactics and communication training;

- assistive listening devices; or

- auditory training (we planned to analyse these second two in separate meta-analyses).

We did not consider studies where the intervention was delivered in a group setting.

\section{Types of outcome measures}

The following outcomes were analysed in the review, but we did not use them as a basis for including or excluding studies. We analysed the data at the trial endpoint, with a planned subgroup analysis to compare different trial endpoints. The ranked hierarchy of instruments was derived from those reported in two systematic reviews (Chisolm 2007 Table 4; Granberg 2014 Table 4).

\section{Primary outcomes}

- Hearing-specific health-related quality of life, where participation was the key domain. This was measured using self-report questionnaires. Where multiple questionnaires were used in a study, we proposed a ranked hierarchy of instruments whereby we identified the primary outcome based on the following in order of importance:

- Hearing Handicap Inventory for the Elderly (HHIE; Ventry 1982) or HHI for Adults (HHIA; Newman 1990), if the HHIE was not used;

- Quantified Denver Scale of Communication (QDS; Tuley 1990);

- Auditory Disability Preference - Visual Analogue Scale (ADPIVAS; Joore 2002); and

- any questionnaire not specified above that was relevant to hearing-specific health-related quality of life.

For example, if both the HHIE and QDS were included in one study, we would use only the HHIE in any meta-analysis.

- Adverse effect: pain. As reported by the patient as pain, discomfort, tenderness or skin irritation, or reported as occurrence of ear infection as a consequence of hearing aid fitting.

\section{Secondary outcomes}

- Health-related quality of life. A ranked hierarchy of self-report outcome measures was proposed in the following order:

- Health Utilities Index Mark 3 (HUI-3; Furlong 2001);

- EQ-5D (Rabin 2001); 
- SF-36 (Ware 1992), or if not reported other short forms of the SF-36;

- Glasgow Benefit Inventory (GBI; Robinson 1996);

- World Health Organization Disability Assessment Schedule (WHO-DAS; WHO 2012b);

- Self-Evaluation of Life Function (SELF; Linn 1984); and

- any questionnaire not specified above that was relevant to health-related quality of life.

- Listening ability. A ranked hierarchy of self-report outcome measures was proposed in the following order:

- Abbreviated Profile of Hearing Aid Benefit (APHAB; Cox 1995);

- Speech, Spatial and Qualities of Hearing (SSQ; Gatehouse 2004);

- Glasgow Hearing Aid Benefit Profile (GHABP, residual disability subscale; Gatehouse 1999); and

- any questionnaire not specified above that was relevant to self-report of listening ability.

- Adverse effect: noise-induced hearing loss, for example due to over-amplification from inappropriate hearing aid fitting.

\section{Search methods for identification of studies}

The Cochrane ENT Information Specialist conducted systematic searches for randomised controlled trials and controlled clinical trials. There were no language, publication year or publication status restrictions. The date of the search was 23 March 2017. We contacted original authors for clarification and further data when trial reports were unclear, and arranged translations of papers where necessary.

\section{Electronic searches}

We searched the following databases from their inception for published, unpublished and ongoing trials:

- the Cochrane Register of Studies Online (searched 23 March 2017);

- Epub Ahead of Print, In-Process \& Other Non-Indexed Citations, Ovid MEDLINE(R) Daily and Ovid MEDLINE(R) (1946 to 23 March 2017);

- Ovid Embase (1974 to 23 March 2017);

- EBSCO CINAHL (1982 to 23 March 2017);

- Ovid Cab (1910 to 23 March 2017);

- LILACS (searched 23 March 2017);

- KoreaMed (searched 23 March 2017);

- PakMediNet (searched 23 March 2017);

- Web of Knowledge, Web of Science (1945 to 23 March 2017);

- ClinicalTrials.gov, www.clinicaltrials.gov (searched via the Cochrane Register of Studies 23 March 2017);

- World Health Organization (WHO) International Clinical Trials Registry Platform (ICTRP), www.who.int/ictrp (searched 23 March 2017);

- Google Scholar (searched 23 March 2017).

In searches prior to 2017 we also searched PubMed 1946 to January 2016 as a top-up to Ovid MEDLINE and IndMed to January 2017.

The Information Specialist modelled subject strategies for databases on the search strategy designed for CENTRAL. Where appropriate, they were combined with subject strategy adaptations of the highly sensitive search strategy designed by Cochrane for identifying randomised controlled trials and controlled clinical trials (as described in the Cochrane Handbook for Systematic Reviews of Interventions Version 5.1.0, Box 6.4.b. (Handbook 2011). Search strategies for major databases including CENTRAL are provided in Appendix 1.

\section{Searching other resources}

We scanned the reference lists of identified publications for additional trials and contacted trial authors where necessary. In addition, the Information Specialist searched Ovid MEDLINE to retrieve existing systematic reviews relevant to this systematic review so that we could scan their reference lists for additional trials.

\section{Data collection and analysis}

\section{Selection of studies}

Material downloaded from electronic sources included details of author, institution, journal of publication and abstract. Two of the three screening authors (MF, DH and FB) independently screened each study against the inclusion criteria to determine their eligibility for inclusion in the review. Where the decision about any one study was not unanimous among the screening authors or there was insufficient information, we acquired the full article for further inspection. Once the full articles were obtained, we decided whether the studies met the review criteria, and where there were disagreements we reached a final decision by discussion and consensus.

We revised the inclusion criteria for participants part-way through the review process. The revised definition for Types of participants and the final decision about which studies to include were subject to independent review by an external expert committee to ensure that it was appropriate for the review question. The risk of revising the protocol was minimised, because this committee also independently evaluated the studies (shortlisted by the authors) and agreed that they met the new inclusion criterion. See Differences between protocol and review for details.

\section{Data extraction and management}

MF and PK independently extracted data from the articles. We recorded the extracted data using the Covidence systematic review software (Covidence 2017) on review-specific forms. We developed and assessed these for suitability through pilot testing prior to independent data extraction. Where there were discrepancies in the data extracted from either the main body or a table we made a final decision by discussion. Where data were extracted from graphs, we used the average of the two independent extractions. Whenever discrepancies were detected, a third author was involved to reconsider and resolve the differences.

Information extracted included: trial design, setting, methods of randomisation and blinding, power, inclusion and exclusion criteria, type of intervention and control, time since hearing aid fitting, duration of follow-up, outcome measures and statistical tests.

For both the intervention and control groups, data extraction included: baseline characteristics of participants (number, sex, age), details of their hearing loss (mean, standard deviation, range), and details of any attrition or exclusion. For the intervention group, 
we extracted details of hearing aids (ear on which they were worn, analogue or digital, in-the-ear or behind-the-ear, manufacturer, unilateral or bilateral hearing aid fittings, and fitting procedures if reported).

Outcome measure data included: group means and standard deviations at pre- and post-intervention and follow-up, number of participants and results of statistical tests of between-group comparisons.

We contacted the authors of all included studies as further information was required on all studies after inspection of the full articles. After independent data extraction by MF and PK, there were no disagreements that could not be resolved through discussion.

\section{Assessment of risk of bias in included studies}

MF and PK independently assessed the risk of bias of the included trials, with the following taken into consideration, as guided by theCochrane Handbook for Systematic Reviews of Interventions (Handbook 2011):

- sequence generation;

- allocation concealment;

- blinding of (i) participants and study personnel (performance bias), and (ii) outcome assessment (detection bias);

- incomplete outcome data;

- selective outcome reporting; and

- other sources of bias.

We used the Cochrane 'Risk of bias' tool in RevMan 5.3 (RevMan 2014), which involved describing each of these domains as reported in the trial and then assigning a judgement about the risk of bias on the outcomes measured as a result of each entry: 'low', 'high' or 'unclear' risk of bias. Where there were disagreements that could not be resolved, DH reviewed the paper and made a final judgement.

\section{Measures of treatment effect}

We expressed continuous outcomes as the size of the difference in treatment effect between an intervention group and a comparator group at the trial endpoint in terms of either the mean difference (MD), when the same outcome measures were used across studies, or the standardised mean difference (SMD), when different outcome measures were used across studies. The calculation of the effect size used the pooled standard deviation, and we reported the $95 \%$ confidence interval (Cl) for each MD or SMD. An effect size less than 0 indicated that a larger treatment effect was observed in the treatment group relative to the comparator group. We did not extract any binary data.

\section{Unit of analysis issues}

The unit of analysis was the participant. We anticipated that participant-level data would not generally be available and therefore reported suitable summary statistics that were provided in the articles. As stated above, in the case of cross-over trials we only included data from the first period.

\section{Dealing with missing data}

We contacted the corresponding authors of all the included studies to obtain missing data, except McArdle 2005 because they used imputation to account for missing data. We obtained participantlevel data from Humes 2017, but we received no data from the other three studies, two of which no longer had the data available (Melin 1987; Mulrow 1990). We either took data from tables presented in the published manuscripts or estimated data from published figures, as described in the Data extraction and management section. Where standard deviations were not reported or provided by the authors, we estimated standard deviations in RevMan 5.3 (RevMan 2014) using available data, such as standard errors, 95\% confidence intervals, $P$ values and $t$ values. We reported the extent of the missing data within studies. Data were not available for any subgroups of interest.

\section{Assessment of heterogeneity}

We assessed heterogeneity among treatment effect sizes using RevMan 5.3 (RevMan 2014), and we expressed this in terms of the $I^{2}$ statistic. We assessed the statistical significance $(P=0.10)$ using a $\mathrm{Chi}^{2}$ test with $\mathrm{K}-1$ degrees of freedom. We quantified heterogeneity in terms of the $\mathrm{I}^{2}$ statistic with low, medium and high ranges of $0 \%$ to $40 \%, 41 \%$ to $60 \%$ and $61 \%$ to $100 \%$, respectively.

\section{Assessment of reporting biases}

We planned to assess publication bias by examining a funnel plot of the size of treatment effects plotted against their variability. However, there were insufficient studies to assess whether the plot was symmetrical or to quantify the deviation from the expected symmetrical pattern using the 'trim and fill' method (Duval 2000).

\section{Data synthesis}

We conducted random-effects meta-analyses of the MDs and SMDs using RevMan 5.3 (RevMan 2014). We used random-effects models as we anticipated significant heterogeneity among treatment effects across studies. For each meta-analysis, we reported a summary effect size estimate in terms of the MD or SMD, together with its $95 \%$ confidence interval. We calculated summary effects using the inverse variance procedure.

To help with the interpretation of data, we estimated the 'equivalent' change on one of the more commonly used scales whenever SMDs were used to summarise data from a few different patient-reported outcome instruments. We multiplied the observed SMD by the standard deviation of a representative study.

\section{Subgroup analysis and investigation of heterogeneity}

We had planned to use subgroup analyses to assess possible sources of heterogeneity. While heterogeneity was identified (see Effects of interventions), it was not possible to perform subgroup analyses based on age, sex and degree of hearing loss as data on subgroups were not available. Time between fitting and trial endpoint was also identified as a possible source of heterogeneity, but a planned subgroup analysis comparing trials with different endpoints (up to three months, over three months to six months and six months or more) was not possible due to the small number of included studies. We conducted a post-hoc subgroup analysis for the hearing-specific health-related quality of life outcome due to the observed pattern of effects across studies (see Effects of interventions) and the differences in participant groups (e.g. military personnel compared with community dwellers). 


\section{Sensitivity analysis}

We had planned to perform sensitivity analyses to informally test the robustness of assumptions from the data. The small number of studies precluded sensitivity analyses to assess uncertainty about aspects of the included studies, in terms of randomisation (random/quasi-random), missing data (greater than 30\% at the primary endpoint) and description of hearing loss (mild/moderate).

\section{GRADE and 'Summary of findings' table}

Using the GRADE approach, two review authors (PK, DH) independently rated the overall quality of evidence using the GDT tool (http://www.guidelinedevelopment.org/) for the main comparison pairs listed in the Types of interventions section. MF and LYC reviewed the ratings and resolved any queries and disagreements. In addition, we also presented the data to a guideline panel that consisted of clinical experts, methodologists and patient representatives and we took their comments into account to reach the final ratings. The quality of evidence reflects the extent to which we are confident that an estimate of effect is close to its true value and we applied this in the interpretation of results. The four possible ratings were: high, moderate, low and very low. A rating of high quality of evidence implies that we are confident in our estimate of effect and that further research is very unlikely to change our confidence in the estimate of effect. A rating of very low quality implies that any estimate of effect obtained is very uncertain.

The GRADE approach rates evidence from RCTs that do not have serious limitations as high quality. However, several factors can lead to the downgrading of the evidence to moderate, low or very low. The degree of downgrading is determined by the seriousness of these factors:

- study limitations (risk of bias);

- inconsistency;

- indirectness of evidence;
- imprecision; and

- publication bias.

We included a 'Summary of findings' table, constructed according to the recommendations described in Chapter 10 of the Cochrane Handbook for Systematic Reviews of Interventions (Handbook 2011). We included the following outcomes in the 'Summary of findings' table: hearing-specific health-related quality of life, health-related quality of life, listening ability and adverse effects.

\section{RES U L T S}

\section{Description of studies}

See: Characteristics of included studies; Characteristics of excluded studies.

\section{Results of the search}

The search identified 4821 records including papers, reviews, conference abstracts and registered clinical trials, of which 2840 remained after removing duplicates. We discarded 2748 records based on the title and/or abstract, which left 92 records. We discarded a further 81 records. Of these, we discarded 69 on the basis that they were not RCTs, 10 because the intervention was not hearing aids alone, one because the control was not appropriate according to the protocol, and one record was an ongoing clinical trial (NCT03002142).

Subsequently, we formally excluded five studies for the reasons given in Excluded studies. An erratum (McArdle 2006) was associated with one of the included studies (McArdle 2005). Five studies were eligible for inclusion in the review (Figure 1). Three studies reported quantitative data that could be included in the meta-analyses (Humes 2017; McArdle 2005; Mulrow 1990). Methodological information was supplemented by other articles on the same study for McArdle 2005 by Chisolm 2005, and for Adrait 2017 by Nguyen 2017. 
Figure 1. Study flow diagram.

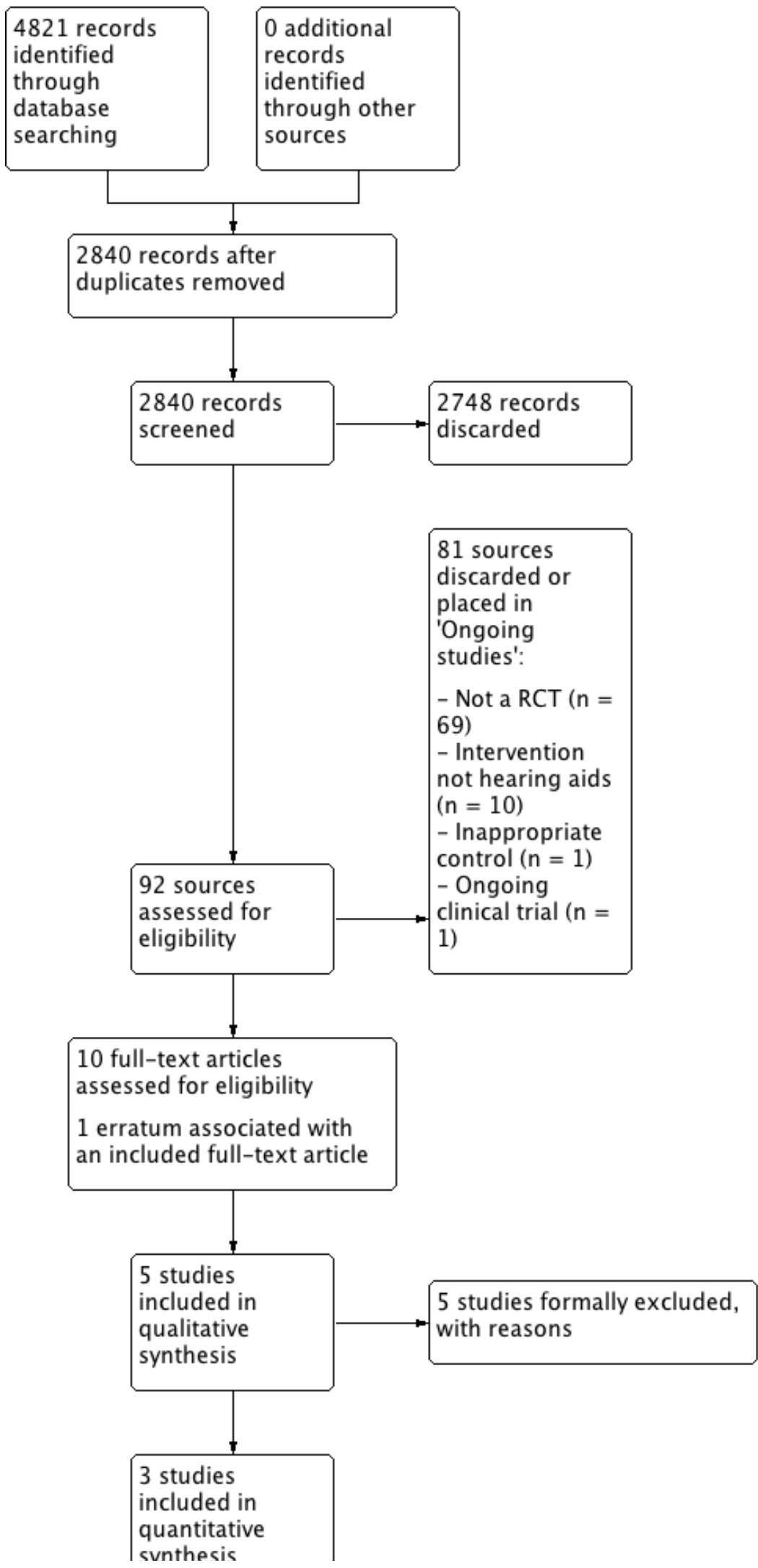


Figure 1. (Continued)

quantitative

synthesis

(meta-analysis)

\section{Included studies}

\section{Design}

All five included studies reported using a randomised controlled (parallel) design. Three studies used a waiting list control group (McArdle 2005; Melin 1987; Mulrow 1990), and the control group in two studies used placebo hearing aids (Adrait 2017; Humes 2017). All five studies specified that the control group received the active hearing aid intervention at the end of the randomised phase, and further follow-up was conducted in all but one study (Mulrow 1990). Participants in the waiting list groups received hearing aids (McArdle 2005; Melin 1987; Mulrow 1990), and the placebo hearing aids were reprogrammed to provide amplification (Adrait 2017; Humes 2017).

The trial endpoint varied across studies with post-hearing aid fitting outcomes obtained at six weeks (Humes 2017; Melin 1987), two months (McArdle 2005), 16 weeks (Mulrow 1990), and six months (Adrait 2017). None of the studies reported long-term outcomes of over one year.

\section{Setting}

Three studies were published between 1987 and 2005 (McArdle 2005; Melin 1987; Mulrow 1990), and two were published in 2017 (Adrait 2017; Humes 2017). Two studies were set in Veterans Association clinics in the USA (McArdle 2005; Mulrow 1990), two in university clinics in the USA and Sweden (Humes 2017; Melin 1987), and one in a hospital setting in France (Adrait 2017).

\section{Population and sample size}

Age, sex and hearing loss were reported at the group level for all studies except Melin 1987, which reported these data at the participant level. The total sample size for the included studies was 825.

All the studies recruited older adult participants, with the mean age in individual studies ranging between 69 and 83 years. Two studies involved military personnel and had almost exclusively male participants (McArdle 2005 98\%; Mulrow 1990 99\%), whereas in the other studies males accounted for between $33 \%$ and $57 \%$ of the samples. All reported mean thresholds were within the mild to moderate hearing loss range of this review's inclusion criteria as described in Types of participants. The frequencies at which mean hearing thresholds were reported varied across studies. All studies had hearing loss as an inclusion criterion but the frequencies and hearing levels used to define the inclusion criteria varied across studies. It is possible that the military veterans were at increased risk of having noise-induced hearing loss. This is typically shown by a notch in the pure-tone audiogram around $4 \mathrm{kHz}$ (i.e. thresholds close to that frequency would be better at adjacent frequencies). There was no evidence of noise-induced hearing loss in the group audiometric results in McArdle 2005, and the presence of noiseinduced hearing loss could not be determined in Mulrow 1990. The participants in Humes 2017, who lived independently in the community, showed no evidence of noise-induced hearing loss in the group audiometric results. The participants in Adrait 2017 all had a diagnosis of Alzheimer's disease. Two studies used normal cognitive function based on the Mini-Mental State Examination as an inclusion criterion (Humes 2017; McArdle 2005), and those with disabling comorbid conditions were excluded from Mulrow 1990.

\section{Interventions and comparisons}

The intervention in each study was acoustic hearing aids, which met the inclusion criteria of this review as described in Types of interventions. Three studies used bilateral hearing fittings (Adrait 2017; Humes 2017; McArdle 2005), and in the two early studies hearing aids were fitted to one ear only in 95\% and $97 \%$ participants respectively (Melin 1987; Mulrow 1990). Two studies reported using in-the-ear hearing aids (McArdle 2005; Mulrow 1990), and three studies used behind-the-ear hearing aids (Adrait 2017; Humes 2017; Melin 1987), with three studies reporting the manufacturer and model of hearing aid used (Adrait 2017; Humes 2017; Melin 1987). Fully digitally programmable hearing aids were used in two studies (Adrait 2017; Humes 2017), with both digital or analogue hearing aids used by McArdle 2005. Humes 2017 used two programming methods for the intervention group. The hearing aids for the Audiology Best practice group were programmed using real-ear measurements according to NAL-NL2, whereas the hearing aids for the Consumer Decides group were preset to three common audiogram configurations, and the participants chose which programme they preferred. The two early studies did not report the technology type (Melin 1987; Mulrow 1990). Three studies used a waiting list comparison group until the first treatment period was completed (McArdle 2005; Melin 1987; Mulrow 1990), and two studies used placebo hearing aids as the comparison (Adrait 2017; Humes 2017), which were programmed to provide no gain so as to be as acoustically transparent as possible.

\section{Outcomes}

Adrait 2017 is one of a series of papers reporting the results of a clinical trial aimed at studying the effects of bilateral hearing aids in patients with age-related hearing loss and Alzheimer's disease (Alzheimer Disease, Presbycusis and Hearing Aids, ADPHA study) on the cognitive, behavioural, quality of life and economic aspects. There were no hearing-specific health-related quality of life or listening ability outcomes measured. Apart from adverse effects, this study did not use any of the outcome measures pre-specified in this review (Types of outcome measures).

\section{Primary outcomes}

Three studies reported hearing-specific health-related quality of life using the HHIE (Humes 2017; McArdle 2005; Mulrow 1990). In addition to the HHIE, Mulrow 1990 also used the Quantified Denver Scale of Communication (QDS). Using the outcome hierarchy defined in Primary outcomes, we only included the HHIE data from Mulrow 1990 in the meta-analyses. 
Only one study measured adverse effects (Adrait 2017); no adverse effects related to pain were reported.

\section{Secondary outcomes}

Health-related quality of life was reported by three studies (Adrait 2017; McArdle 2005; Mulrow 1990). Two studies used outcome measures that were generic to the clinical population being assessed: McArdle 2005 used the WHO-DAS II and Mulrow 1990 used the Self-Evaluation of Life Function (SELF).

Adrait 2017 used a disease-specific health-related quality of life measure for patients with Alzheimer's disease (Alzheimer's Disease Related Quality of Life, ADRQL; Rabins 1999). Data for the ADRQL were collected from the caregivers, rather than the patients. Therefore, this was not an outcome measure of interest prespecified in this review.

Listening ability was reported in three studies. Humes 2017 used the Profile of Hearing Aid Performance (PHAP; Cox 1990), McArdle 2005 used the Abbreviated Profile of Hearing Aid Benefit (APHAB) and Melin 1987 used a hearing ability scaling assessment. We did not include the hearing ability scaling assessment, which is not a validated measure, in the meta-analysis as not all the data required were available in the paper and were no longer available.

Only one study measured adverse effects (Adrait 2017); no adverse effects related to noise-induced hearing loss were reported.

\section{Excluded studies}

Details of the five studies that we excluded after clarification of the methods used are shown in Characteristics of excluded studies.

\section{Risk of bias in included studies}

Two authors (MF, PK) critically reviewed the methodology of the included studies. We contacted the authors from all five included studies to further establish aspects of risk of bias that were unclear from the full article. We received responses from four authors (Adrait 2017; Humes 2017; McArdle 2005; Melin 1987). The 'Risk of bias' summary for the included studies is shown in Figure 2. One study showed a low risk of bias in six categories (Adrait 2017), two studies showed a low risk of bias in four categories (Humes 2017; Mulrow 1990), and two studies showed a low risk of bias in three categories (McArdle 2005; Melin 1987).The 'Risk of bias' graph presented as percentages across all studies is shown in Figure 3. Further details are described below. 
Figure 2. 'Risk of bias' summary: review authors' judgements about each risk of bias item for each included study.

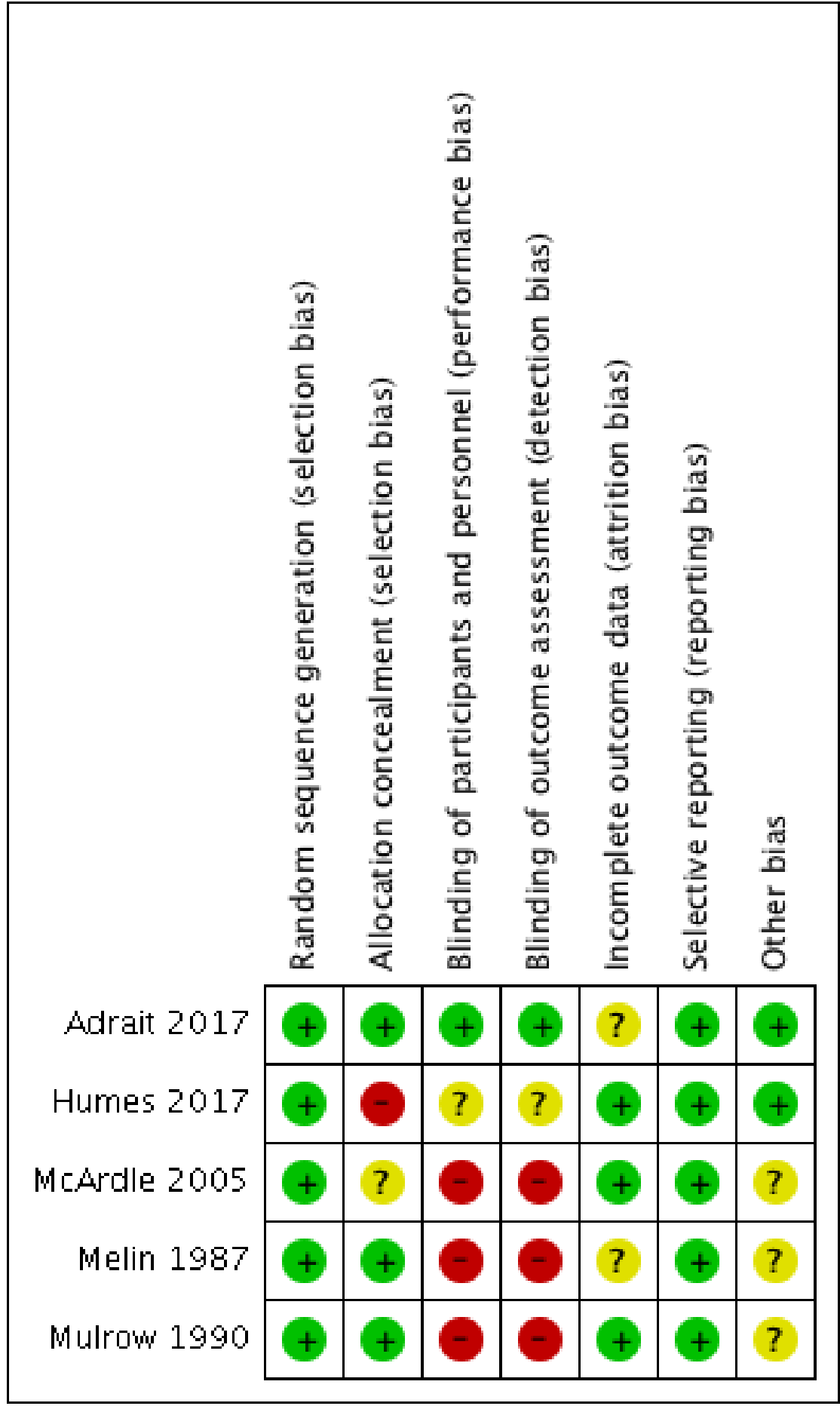


Figure 3. 'Risk of bias' graph: review authors' judgements about each risk of bias item presented as percentages across all included studies.

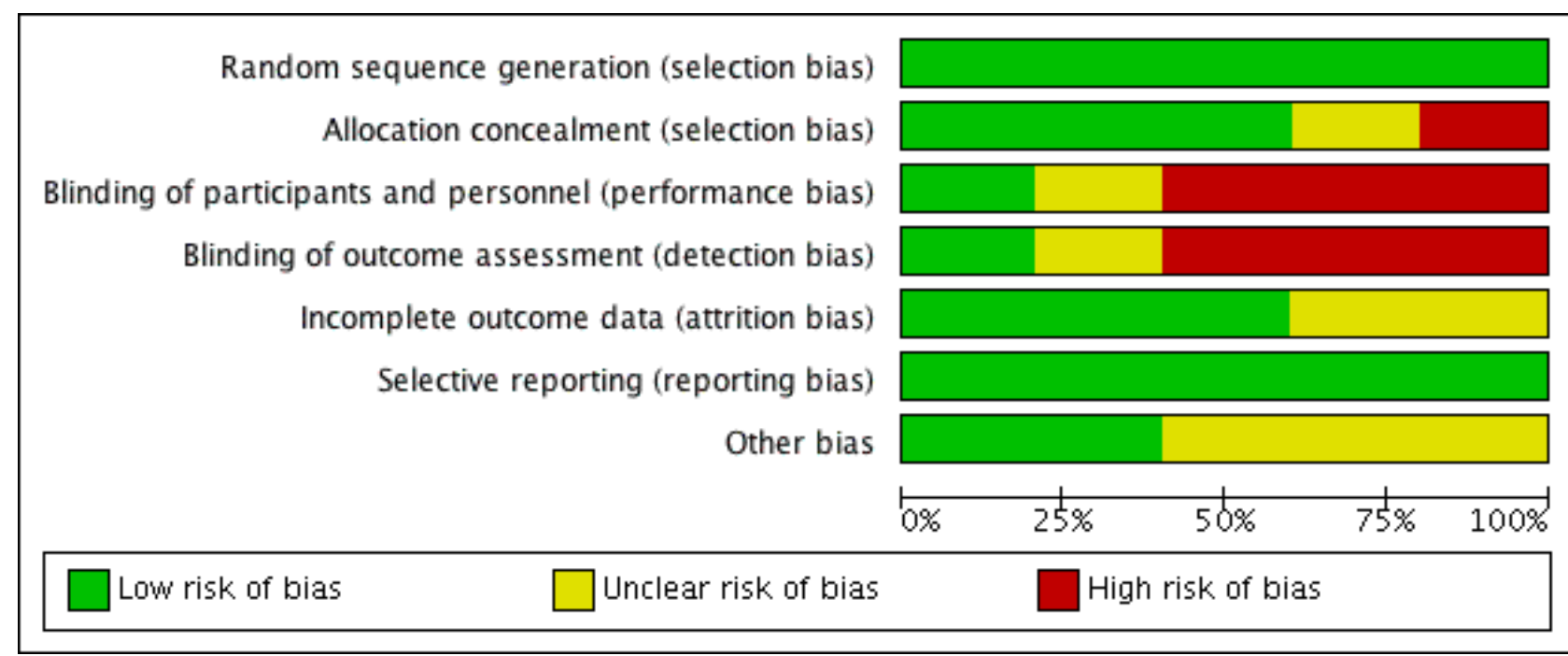

Allocation

\section{Random sequence generation}

We judged all five included studies to be at a low risk of bias due to sequence generation.

\section{Allocation concealment}

We judged three studies to be at low risk of bias due to allocation concealment as a remote allocation service or sealed, opaque envelopes were used (Adrait 2017; Melin 1987; Mulrow 1990). There was insufficient information to make a judgement for McArdle 2005, and we judged Humes 2017 to be at high risk of bias because the randomisation list was visible to the clinical trial co-ordinator who also allocated participants to the treatment or comparator group.

\section{Blinding}

Until recently, the nature of hearing aids as an intervention has led to substantial difficulty in designing trials that aim to control for performance bias. In addition, the visibility of hearing aids increases the risk of detection bias when a researcher measures outcomes. Thus, we judged all the studies published during or before 2005 that used waiting list controls to be at high risk for both performance and detection bias (McArdle 2005; Melin 1987; Mulrow 1990). The use of placebo hearing aids allows blinding of participants and personnel and blinded assessment of outcomes if they are visibly identical to active hearing aids and the fitting procedure for active and placebo devices is indistinguishable to the participant. These criteria were met by Adrait 2017, which we judged to be at low risk of performance and detection bias. The Humes 2017 study also used placebo devices but we judged this to have an unclear risk of performance and detection bias as there was potential for participants to be unblinded due to contact with the clinical trial co-ordinator who was not blinded to allocation.

\section{Incomplete outcome data}

We judged attrition bias to be low for three studies (Humes 2017; McArdle 2005; Mulrow 1990), as dropouts were clearly reported and dropout numbers were low and were equal across both the intervention and comparator groups. The reasons for dropouts were generally not related to the intervention. McArdle 2005 used imputation to account for missing data due to dropouts. There was insufficient information about the other two studies to assess the risk of bias (Adrait 2017; Melin 1987), which led to a judgement of unclear risk.

\section{Selective reporting}

We judged all five included studies to be at low risk of selective reporting. Two studies published study protocols and reported the stated outcomes (Adrait 2017; Humes 2017). Although no protocols had been published prior to the completion of two of the older studies (McArdle 2005; Mulrow 1990), the reported outcomes followed the rationale of the studies. Contact with the authors of Melin 1987 indicated that all outcomes measured had been included.

\section{Other potential sources of bias}

Three of the included studies had the comparator group on a waiting list (McArdle 2005; Melin 1987; Mulrow 1990), and all of these studies had specified that all patients would receive hearing aids at the end of the randomised phase. Studies using waiting list controls have a risk of overestimating the benefit of an intervention, especially if participants perceived that the reported outcomes could determine their eligibility for an intervention (Furukawa 2014). As all the participants were aware that they would receive the interventions at the end of the study, we thought that the risks were less clear in this case (unclear risk of bias).

\section{Effects of interventions}

See: Summary of findings for the main comparison Hearing aids versus no hearing aids for mild to moderate hearing loss in adults

See: Summary of findings for the main comparison.

For the McArdle 2005 study, we extracted data from Table 3, except the Hearing Handicap Inventory for the Elderly (HHIE) data from the control group at the two-month follow-up, which we took from the table in the Erratum (McArdle 2006). Humes 2017 did not report 
the six-week follow-up results, so we obtained these from the study dataset that was sent at our request. We directly extracted data from Mulrow 1990. Data from Melin 1987 and Adrait 2017 were not included in the meta-analysis (see Characteristics of included studies).

\section{Comparison: hearing aids versus no hearing aids}

\section{Primary outcome measures}

\section{Hearing-specific health-related quality of life}

The HHIE at study endpoint was used to measure hearing-specific health-related quality of life in all three studies included in a metaanalysis. Mean differences (MDs) and their $95 \%$ confidence intervals (Cls) are shown in Analysis 1.1 (Figure 4).

Figure 4. Forest plot of comparison: 1 Hearing aids versus no/placebo hearing aids, outcome: 1.1 Hearing-specific health-related quality of life. Assessed using Hearing Handicap Inventory for the Elderly (HHIE) in all studies.

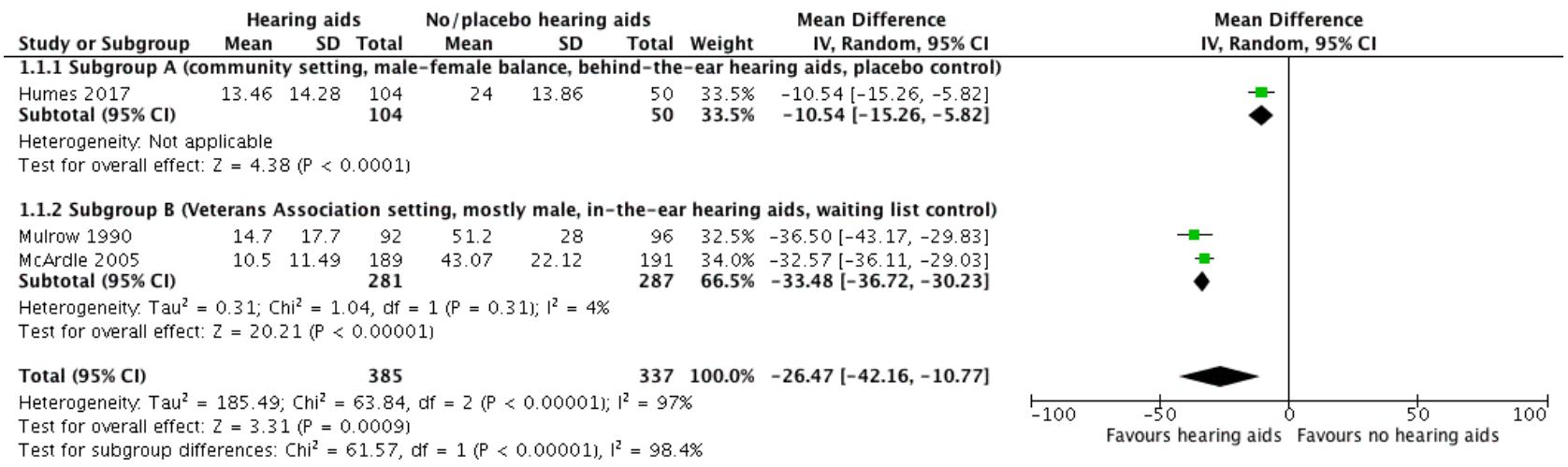

The random-effects meta-analysis showed a significant overall effect that favoured hearing aids over the unaided/placebo comparison (scale range 0 to 100 ) (MD $-26.47,95 \% \mathrm{Cl}-42.16$ to $-10.77 ; 722$ participants; three studies) (moderate-quality evidence) (Analysis 1.1). We observed considerable heterogeneity $(12=$ $97 \%)$, which was statistically significant $\left(\mathrm{Chi}^{2}=63.84\right.$, df $=2$, $\mathrm{P}<0.00001$ ). We conducted an unplanned subgroup analysis (Veterans Association setting, mostly male, in-the-ear hearing aids, waiting list control versus community setting, male-female balance, behind-the-ear hearing aids, placebo control study) to explore this statistical heterogeneity. This showed statistically significant subgroup effects $(P<0.00001)$. The MD was -33.48 , (95\% Cl -36.72 to -30.23 ; 568 participants; two studies; $\left.\right|^{2}=4 \%$ ) for the veterans subgroup (McArdle 2005; Mulrow 1990), and -10.54 (95\% Cl -15.26 to $-5.82 ; 154$ participants) for the community setting subgroup (Humes 2017).

The Quantified Denver Scale of Communication (QDS) results from Mulrow 1990 were not included in the meta-analysis as our protocol specified a hierarchy of inclusion in which the HHIE had a higher priority. However, the QDS also indicated a significant effect favouring hearing aids (scale range 0 to 100 ) (MD -26.5, 95\% Cl -33.6 to $-19.4 ; 188$ participants).

\section{Adverse effects}

Only one study ( $n=48)$ measured adverse effects (Adrait 2017), and no adverse effects related to pain were reported (very low-quality evidence).

\section{Secondary outcome measures}

\section{Health-related quality of life}

Health-related quality of life at study endpoint was measured using the World Health Organization Disability Assessment Schedule (WHO-DAS) II (McArdle 2005) and the Self-Evaluation of Life Function (SELF) (Mulrow 1990). A random-effects meta-analysis showed a significant overall effect that favoured hearing aids over the unaided/placebo comparison (standardised mean difference (SMD) $-0.38,95 \% \mathrm{Cl}-0.55$ to $-0.21 ; 568$ participants; two studies, $\mathrm{I}^{2}=$ $6 \%$ ) (moderate-quality evidence) (see Analysis 1.2; Figure 5). 
Figure 5. Forest plot of comparison: 1 Hearing aids versus no/placebo hearing aids, outcome: 1.2 Health-related quality of life.

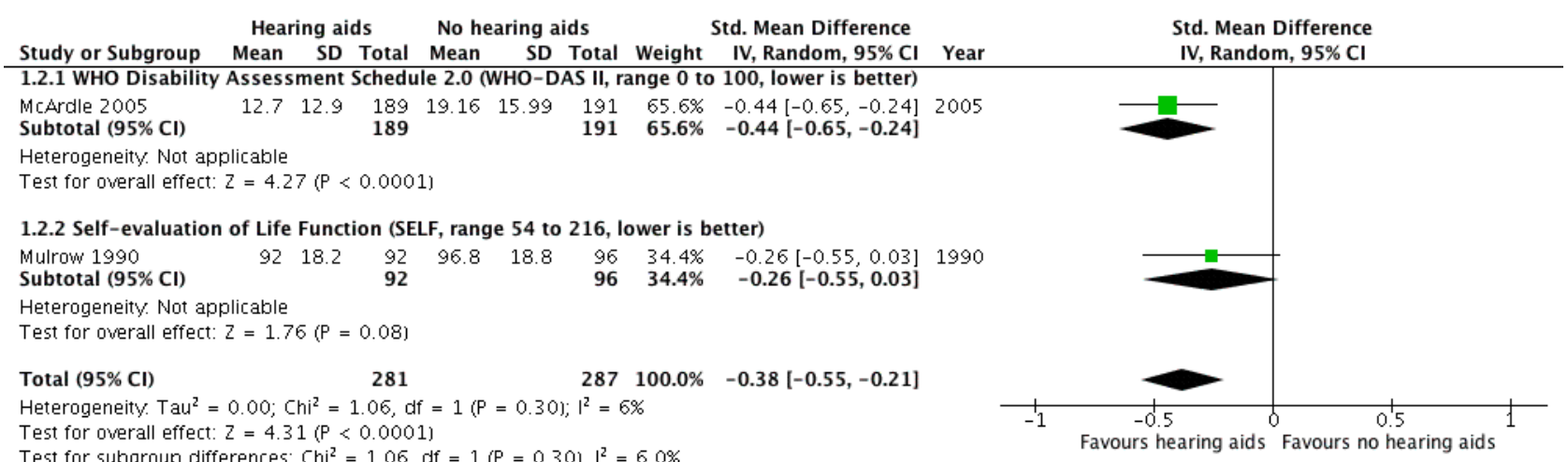

\section{Listening ability}

Listening ability at study endpoint was measured using the Profile of Hearing Aid Performance (PHAP) in Humes 2017 and the Abbreviated Profile of Hearing Aid Benefit (APHAB) in McArdle 2005. $A$ random-effects meta-analysis showed a significant overall effect that favoured hearing aids over the unaided/placebo comparison (SMD $-1.88,95 \% \mathrm{Cl}-3.24$ to $-0.52 ; 534$ participants; two studies) (moderate-quality evidence) (Analysis 1.3; Figure 6). We observed considerable heterogeneity $(12=97 \%)$, which was statistically significant $\left(\mathrm{Chi}^{2}=31.12, \mathrm{df}=1, \mathrm{P}<0.00001\right)$. The unplanned subgroup analysis showed statistically significant $(P<0.00001)$ subgroup effects, where McArdle 2005 had a SMD of $-2.57(95 \% \mathrm{Cl}$ -2.84 to $-2.30 ; 380$ participants) and Humes 2017 had a SMD of -1.18 (95\% $\mathrm{Cl}-1.54$ to $-0.81 ; 154$ participants).

\section{Figure 6. Forest plot of comparison: 1 Hearing aids versus no/placebo hearing aids, outcome: 1.3 Listening ability.}

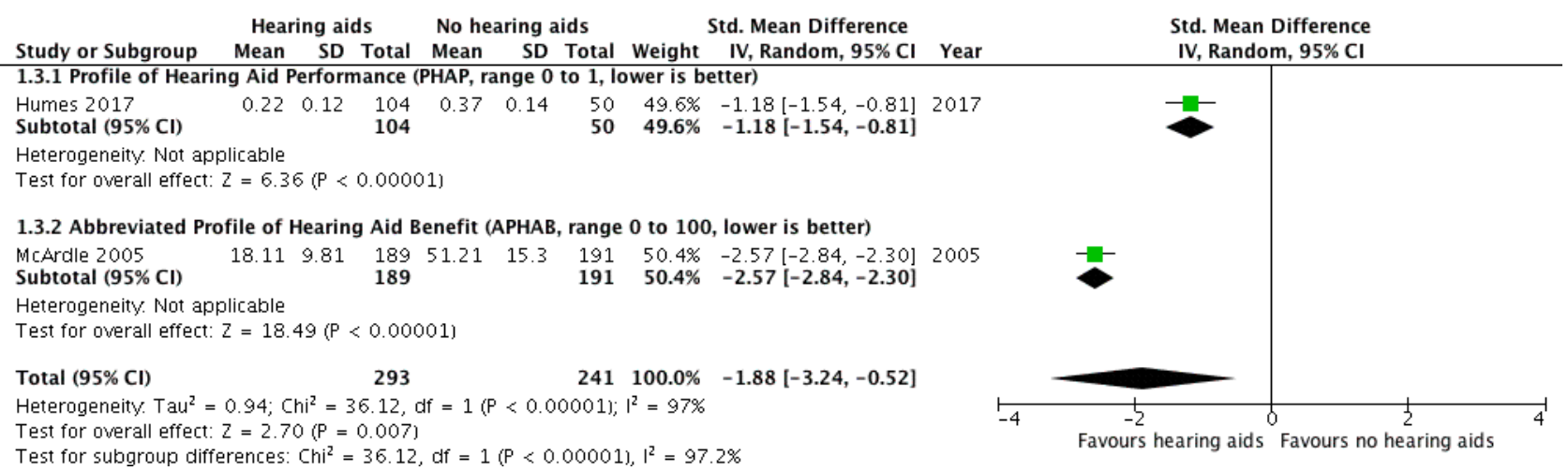

\section{Adverse effects}

Only one study ( $n=48$ ) measured adverse effects (Adrait 2017), and no adverse effects related to noise-induced hearing loss were reported (very low-quality evidence).

\section{DISCUSSION}

\section{Summary of main results}

For hearing-specific health-related quality of life where participation is the key domain (primary outcome measure), we found evidence that hearing aids had a large beneficial effect in reducing participation restrictions. Our confidence in the quality of the evidence for hearing-specific health-related quality of life was moderate due to high risk of bias (see Summary of findings for the main comparison). Significant differences in the size of effects were apparent across studies; the effects reported by the two individual Veterans Association (VA) studies were similar (McArdle 2005; Mulrow 1990), and more than twice the size of the effect reported by Humes 2017. However, all three studies individually reported large effects (standardised mean difference $(S M D)>0.70$ ) that favoured hearing aids, meaning that while further evidence may change the size of the overall effect on hearing-specific healthrelated quality of life, we have high confidence in the magnitude and direction of the effect.

For health-related quality of life (secondary outcome measure) there was evidence of a small beneficial overall effect of hearing aids compared to the unaided/placebo condition. Two different outcome measures were used. One study reported a significant benefit of hearing aids compared to the unaided/ placebo condition using the World Health Organization Disability Assessment Schedule (WHO-DAS II) (SMD -0.44, 95\% confidence interval ( $\mathrm{Cl})-0.65$ to $-0.24 ; 380$ participants), and another study reported no significant effect of hearing aids using the SelfEvaluation of Life Function (SELF) (SMD $-0.26,95 \% \mathrm{Cl}-0.55$ to 0.03 ; 188 participants). Heterogeneity was low. Our confidence in the quality of the evidence for health-related quality of life was moderate due to high risk of bias (see Summary of findings for the main comparison). 
For listening ability (secondary outcome measure), there was a large beneficial effect of hearing aids when compared to the unaided/placebo condition. The two studies used different outcome measures with one, Abbreviated Profile of Hearing Aid Benefit (APHAB), being an abbreviated version of the other (PHAP). Both measures revealed large beneficial effects that favoured the use of hearing aids. Our confidence in the quality of the evidence for listening ability was moderate due to high risk of bias (see Summary of findings for the main comparison), although we have high confidence that the effect was both large and beneficial.

Adverse effects associated with hearing aids were measured in only one study but no adverse effects were reported that related to pain or noise-induced hearing loss, so it was not possible to comment on the reported benefits against harms.

\section{Overall completeness and applicability of evidence}

Five randomised controlled trials (RCTs) met the inclusion criteria for this review. Three studies assessed hearing-specific healthrelated quality of life using measures that fit within our pre-defined hierarchy of self-report outcome measures, of which one measure (the Hearing Handicap Inventory for the Elderly, HHIE) was used in all three studies. High heterogeneity and subgroup differences for the HHIE results stem from the Humes 2017 study of older adults living in the general community, in which a smaller effect was observed than was found in the two VA studies (McArdle 2005; Mulrow 1990). The participants in the Humes 2017 study had a more even male-female split (57:43), had better average hearing thresholds, were provided with behind-the-ear hearing aids for a cost, and the comparison group received placebo hearing aids. In contrast, the participants in the VA studies were almost exclusively male veterans who may have experienced gunfire or explosive noise that may have resulted in noise-induced hearing loss and the possibility of compensation claims, had poorer hearing, were provided with in-the-ear hearing aids at no cost, and were compared to a waiting list (passive control) group. These participant and methodological differences were likely to account for the differences in the estimated size of the effects between the studies for both the hearing-specific health-related quality of life and listening ability outcomes. As there were numerous differences between these two groups of participants (military veterans versus community dwellers), we considered whether it was appropriate to pool the studies. On the basis that recommendations for the clinical management of hearing loss using hearing aids would not differ between these two groups, we concluded that the data from the studies should therefore be pooled. The choice of outcome measure to assess health-related quality of life was inconsistent, although heterogeneity in the reported effects was low. The WHODAS II used in the McArdle 2005 study has since been superseded by the WHO-DAS 2.0 (WHO 2012b), which directly links to the International Classification of Functioning, Disability and Health (ICF) framework.

We did not include cognition as an outcome in the protocol because of the wide variety and uncertainty of definitions of specific domains within the broader context of cognition (e.g. attention, memory, processing speed). The Short Portable Mental Status Questionnaire and Geriatric Depression Scale measures reported in one included study (Mulrow 1990), alongside a raft of measures related to Alzheimer's disease in another study (Adrait 2017), were not specified in the protocol, and we did not analyse or report the results. Mulrow 1990 reported significant improvements in the hearing aid group on both measures, whereas there were no group differences for any of the measures reported by Adrait 2017.

The planned subgroup analyses (age, sex and degree of hearing loss) could not be performed as data from these subgroups were not reported. Outcomes were measured for short-to-medium term follow-up only (six weeks to six months), with no studies reporting long-term outcome at more than one year for either intervention or control groups separately. In terms of completeness, there are a limited number of RCTs, a preponderance of participants who were men who had poorer hearing, inconsistency in the choice of outcome measures to assess health-related quality of life and listening ability, and inconsistency in the choice of comparator (waiting list versus placebo control).

In terms of applicability, all participant samples were reported to have mild to moderate hearing loss. The three studies included in the meta-analyses represented the majority of the participants from the included studies $(n=738 ; 89 \%)$. However, two of these study samples $(\mathrm{n}=574)$ were overwhelmingly male military veterans (97\% and 99\%) who received their hearing aids free of charge, although there is evidence that the price of hearing aids does not affect outcomes (Humes 2017). Generalisability to other non-military populations that would certainly include a greater proportion of women represents a limitation, which these studies acknowledged, and may reflect the size of the effects on hearingspecific health-related quality of life and listening ability. There was also variability in whether hearing aids were fitted unilaterally or bilaterally. Three of the studies had exclusion criteria that were specific to other comorbid conditions, such as cognitive impairment (two studies specifically screened for this using the Mini-Mental State Examination), which could also limit applicability of the findings to general clinical populations, such as those with dementia. Although Adrait 2017 reported no effect of hearing aids in their population of patients with Alzheimer's disease, the outcomes in that study differed to those specified in our review.

Finally, only two of the five included studies set out specifically to examine whether hearing aids improved quality of life in those who had hearing loss (Adrait 2017; Mulrow 1990), but one of these recruited only people with Alzheimer's disease. The primary aim of McArdle 2005 was to assess the properties of the WHO-DAS II as an outcome measure by examining its responsiveness to the effects of hearing aids. The Melin 1987 study was primarily designed to examine hearing aids as an intervention for people with tinnitus, and the Humes 2017 study focused on different service delivery models.

\section{Quality of the evidence}

We considered the quality of evidence for the primary outcome measure (hearing-specific health-related quality of life) and secondary outcome measures (health-related quality of life and listening ability), as assessed by the GRADE system, to be moderate. We downgraded the quality of the evidence by one level because all studies contributing data to these outcomes had either a rating of unclear and/or high risk bias in at least one of the domains of selection bias, performance and/or detection bias (see Summary of findings for the main comparison).

All five included studies were RCTs. We judged three studies to be at high risk of performance and detection bias (McArdle 2005; Melin 1987; Mulrow 1990). These biases are recognised widely to 
be problematic in hearing aid intervention studies as blinding of patients and assessors can be difficult to achieve. Although Humes 2017 used a placebo hearing aid comparison to control for these biases, the potential for unblinding led to our judgements of unclear risk. However, the use of placebo hearing aids with zerogain prescriptions adopted by both Adrait 2017 and Humes 2017 does demonstrate that it is now possible to blind participants and outcome assessors in hearing aid trials.

We considered downgrading the evidence for the effects of hearing aids on hearing-specific health-related quality of life and listening ability due to both inconsistency and indirectness. There were concerns over inconsistency because of the observed statistical heterogeneity between VA studies (McArdle 2005; Mulrow 1990) and the study of Humes 2017 conducted in the community setting. Concerns arose over indirectness both because the analyses included data from male military veterans and also because of the short follow-up period in Humes 2017 (six weeks). We took the decision not to downgrade due to either inconsistency or indirectness as all studies individually reported large beneficial effects of hearing aids on both outcomes, regardless of whether they had been conducted in the military or community settings and regardless of the duration of follow-up. Thus, the observed heterogeneity did not reduce our confidence in the estimates of these effects.

Finally, we considered downgrading the evidence for healthrelated quality of life due to indirectness. Both studies included in the analysis had samples that were almost exclusively male military veterans (McArdle 2005; Mulrow 1990). However, we did not downgrade the evidence because the estimates of the effect were similar across the two studies and heterogeneity was low despite numerous other differences in their samples and designs (i.e. different outcome measure, follow-up period and hearing aid style and number fitted). Our confidence in the size of the effect was therefore not reduced.

Limitations of the evidence reviewed were the numerous methodological and sampling differences between the two veteran studies (McArdle 2005; Mulrow 1990) and the other included studies, and the lack of long-term outcomes (i.e. greater than one year). There were also inconsistencies in the reporting of the studies and clarification on methodological details had to be sought from the authors of all five studies, with four providing information that was not published. Two of the studies were published before the 2001 CONSORT guidelines yet only one out of the other three studies that could have feasibly been able to follow these guidelines reported the study to that standard (Humes 2017).

\section{Potential biases in the review process}

The electronic searches for this review were comprehensive, with a second search carried out within two months of submission to ensure an up-to-date review. The protocol defined the review process and the roles of the authors, and each source was independently reviewed by two authors. Inclusion did not require specific outcome tools.

We revised the inclusion criteria relating to the types of participants due to concerns that the original criteria stated in the protocol had the potential to bias the study selection process (see Differences between protocol and review).
Although we revised the inclusion criteria for Types of participants part-way through the review, we did this with the important rationale of avoiding a potential bias where studies that were reported in less detail (e.g. group mean hearing threshold data or qualitative descriptions) were more likely to be included than those studies that either reported in more detail or provided us with participant-level data. To minimise the risk of bias in this process, the revised definition for Types of participants and the final decisions about which studies were eligible for inclusion in the review were subject to independent review by an external expert committee to ensure that this was appropriate for the review question.

\section{Agreements and disagreements with other studies or reviews}

This review is consistent with the conclusions of a previous systematic review on hearing aids (Chisolm 2007). Both reviews conclude that hearing aids improve hearing-specific health-related quality of life compared to no hearing aids. However, whereas Chisolm 2007 identified a lack of evidence for more general effects on health-related quality of life in people with hearing loss, in our review we showed a small beneficial effect. These reviews differ in a number of methodological details. The current review includes only randomised controlled trials, whereas Chisolm 2007 included randomised and non-randomised trials. Chisolm 2007 used the Scottish Intercollegiate Guideline Network (SIGN) grading system (Harbour 2001), and included two studies that met the highest level of evidence (level 1, RCTs) (Mulrow 1990; Yueh 2001). We did not include the Yueh 2001 study as the randomisation took place after the participants had been allocated to either hearing aids or no hearing aids on the basis of whether they were eligible to receive hearing aids or not. Our review included the McArdle 2005 study, which had not been published at the time the Chisolm 2007 review was conducted, and we included the study by Melin 1987. The other main differences between the reviews were that our review was able to include three generally well-designed RCTs in the metaanalyses and used a pre-defined hierarchy of outcome measures for each outcome domain.

A more recent review on the benefit of hearing aids for patients with mild sensorineural hearing loss concludes that there is evidence that hearing aids benefit adults with mild sensorineural hearing loss (Johnson 2016). None of the studies included in this review were RCTs, and so we cannot compare the results and conclusions of that review with ours.

\section{AUTHORS' CONCLUSIONS}

\section{Implications for practice}

There is evidence of moderate quality that hearing aids improve participation, overall health-related quality of life and listening ability in adults with mild to moderate hearing loss. If the goals and needs of an individual with hearing loss are to improve their listening abilities and, in doing so, improve their participation with others in everyday life and their health-related quality of life more generally, then hearing aids are an appropriate intervention. The evidence is generally compatible with the widespread provision of hearing aids as the first-line clinical management option in those individuals who seek help. Evidence not considered by this review also suggests that self-management of hearing aids should be considered alongside clinical management to improve 
hearing-related participation and communication over the short term (Barker 2016), and increase hearing aid use (Ferguson 2016).

In light of an absence of evidence for the long-term effects of hearing aids on any of the reported outcome measures, outcomes should be monitored up to at least one year post-fitting to determine whether short-term effects are sustained, and whether additional intervention may be required. The fact that hearing aids are in widespread use as an intervention for hearing loss means that questions about long-term effects could be usefully addressed by a synthesis of evidence from large-scale cohort studies. Such a synthesis would be enabled through the consistent collection and reporting of outcomes at consistent time points following hearing aid fitting.

\section{Implications for research}

Well-designed trials are needed to establish whether the effects of hearing aids vary according to age, gender, type of hearing aid, and degree and type of hearing loss. Trial populations should be sampled to be representative of typical first-time hearing aid recipients in terms of gender, age and hearing thresholds. Trial sample sizes need to be large enough to allow subgroup analysis (e.g. comparison of men and women) and to identify the extent of effects of hearing aids within specific patient groups. The reporting of trials also needs to be sufficiently detailed to permit the extraction of data within such subgroups. Trial sample sizes need to be large enough to achieve sufficient statistical power to detect the minimal clinically important difference (MCID) in the primary outcome measure. However, further research is needed to determine the MCID on outcome measures relevant to hearing aids as there is currently an absence of such information. Fitting hearing aids to standard fitting protocols and hearing aid prescriptions (e.g. NAL-NL2) using probe tube microphones to obtain well-fitted hearing aids is also necessary to ensure hearing aids are functioning as intended.

The choice of outcome measure for assessing the effects of hearing aids on broader aspects of health should be considered carefully. Generic measures of health that include relevant domains such as communication (e.g. WHO-DAS 2.0) may be more likely to show effects of hearing aids than measures that do not include such domains. To demonstrate cost-effectiveness of hearing aid provision, and for comparison against other healthcare conditions and interventions, the use of preference-based instruments should also be considered. These measures need to be selected based on evidence for their validity in evaluating hearing-related interventions and their sensitivity to hearing-related changes in health-related quality of life. For example, the Health Utilities Index Mark III (HUI-3) has been shown to be more sensitive to improvements in quality of life resulting from hearing aids compared to both the EQ-5D and the SF-36, which are less sensitive to hearing-related changes in health (Barton 2004; Davis 2007; Joore 2003).

Greater consistency in the choice of outcome measures across studies would enable direct comparisons of the effects of hearing aids and facilitate meta-analyses. The development of a core outcome set for use in auditory rehabilitation research would not only encourage consistency in the measurement and reporting of outcomes (Barker 2016); if selected through an appropriate process, it would also ensure that outcomes are meaningful to patients and clinicians. There is also a need for greater consistency in the time points at which patients are followed up after hearing aid fitting, with more emphasis on longer-term follow-up outcomes measured at greater than one year.

Measures of cognition should be included in future review updates with a proposed hierarchy based on publications from current ongoing systematic reviews (e.g. Loughrey 2015) and expert consensus (e.g. Pichora-Fuller 2016). The reporting of adverse effects in our included studies was limited and inconsistent and therefore should be given specific attention in the design and reporting of future hearing aid trials. Hearing aid takeup and use was not an outcome in this review as we were specifically examining the difference between the aided and unaided conditions. However, there is value in obtaining a metric for hearing aid use as a secondary measure in future studies in any aided groups. This should be based on a relevant model of health behaviour to help determine behaviours that effectively promote hearing aid use, and how it might be improved (Barker 2016; Coulson 2016).

Further studies should be designed to minimise the risk of performance and detection bias that is inherent in most hearing aid trials. Blinding the patient to the intervention is difficult to achieve with hearing aids but can be achieved by programming hearing aids to provide no amplification, which may be acceptable where there is genuine equipoise around their effectiveness. However, care must still be taken to ensure that such placebo hearing aids cannot be distinguished from active hearing aids based on either their visual appearance or the manner in which they are fitted. Blinding of outcome assessors should be incorporated into the design of future trials and particular care should be taken to ensure both patient and outcome assessor remain blinded when collecting patient-reported outcomes. For example, self-reported outcome data could be obtained via telephone rather than through self-completion of questionnaires on paper or through interview; telephone administration has the additional benefit of increasing the test-retest reliability of patient-reported outcome measures (Weinstein 1997).

Reporting of all future trials should follow the CONSORT guidelines (CONSORT 2010).

\section{ACKN OWLEDGEMENTS}

This project was supported by the National Institute for Health Research, via Cochrane Infrastructure, Cochrane Programme Grant or Cochrane Incentive funding to Cochrane ENT. The views and opinions expressed therein are those of the authors and do not necessarily reflect those of the Systematic Reviews Programme, NIHR, NHS or the Department of Health.

We acknowledge Helen Henshaw who helped to conceive the review question. We would also like to acknowledge Saoussen Ftouh (National Guideline Centre) for methodological support and advice. We thank the Cochrane ENT team for their invaluable help: Samantha Faulkner who designed the search strategy, Martin Burton for strategic advice and Jenny Bellorini for her support and substantial general advice. Finally, we thank the authors of included studies for their willingness to share information and respond to our queries. 


\section{RE F E R E N C E S}

\section{References to studies included in this review}

Adrait 2017 \{published and unpublished data\}

* Adrait A, Perrot X, Nguyen MF, Gueugon M, Petitot C, Collet $L$, et al. Do hearing aids influence behavioral and psychological symptoms in hearing impaired Alzheimer's Disease patients and their caregivers?. Journal of Alzheimer's Disease 2017;58(1):109-21. [DOI: 10.3233/JAD-160792]

Humes 2017 \{published and unpublished data\}

* Humes LE, Rogers S, Quigley TM, Main AK, Kinney DL, Herring $C$. The effects of service-delivery model and purchase price on hearing aid outcomes in older adults: a randomized double-blind placebo-controlled clinical trial. American Journal of Audiology 2017;26(1):53-79.

\section{McArdle 2005 \{published and unpublished data\}}

* McArdle R, Chisolm TH, Abrams HB, Wilson RH, Doyle PJ. The WHO-DAS II: measuring outcomes of hearing aid intervention for adults. Trends in Amplification 2005;9(3):127-43.

Melin 1987 \{published and unpublished data\}

Melin L, Scott B, Lindberg P, Lyttkens L. Hearing aids and tinnitus - an experimental group study. British Journal of Audiology 1987;21(2):91-7.

Mulrow 1990 \{published data only (unpublished sought but not used)\}

Mulrow CD, Aguilar C, Endicott JE, Tuley MR, Velez R, Charlip WS, et al. Quality-of-life changes and hearing impairment: a randomized trial. Annals of Internal Medicine 1990;113(3):188-94.

\section{References to studies excluded from this review}

\section{Abrams 2002 \{published data only\}}

Abrams HB, Hnath-Chisolm T, Guerreiro SM, Ritterman S. The effects of intervention strategy on self-perception of hearing handicap. Amplification and Aural Rehabilitation 1992;5:371-7.

\section{Jerger 1992 \{published data only\}}

Jerger J, Chmiel R, Florin E, Pirozzolo F, Wilson N. Comparison of conventional amplification and an assistive listening device in elderly persons. Ear and Hearing 1996;17(6):490-504.

\section{Lavie 2015 \{published data only\}}

Lavie L, Banai K, Kami A, Attias J. Hearing aid-induced plasticity in the auditory system of older adults: evidence from speech perception. Journal of Speech Language Hearing Research 2015;58:1601-10.

\section{Tolson 2002 \{published data only\}}

Tolson D, Swan I, Knussen C. Hearing disability: a source of distress for older people and carers. British Journal of Nursing 2002;11(15):1021-5.

Yueh 2001 \{published data only\}

Yueh B, Souza PE, McDowell JA, Collins MP, Loovis CF, Hedrick SC, et al. Randomized trial of amplification strategies. Archives of Otolaryngology--Head \& Neck Surgery 2001;127:1197-204.

\section{References to ongoing studies \\ NCT03002142 \{published data only\}}

NCT03002142. Auditory rehabilitation with hearing aids and cognition in Alzheimer patients. https://clinicaltrials.gov/ct2/ show/NCT03002142 (first received 16 March 2016).

\section{Additional references}

\section{Aazh 2015}

Aazh H, Prasher D, Nanchahal K, Moore BCJ. Hearing-aid use and its determinants in the UK National Health Service: a cross-sectional study at the Royal Surrey County Hospital. International Journal of Audiology 2015;54:152-61.

\section{Akeroyd 2014}

Akeroyd MA, Foreman K, Holman JA. Estimates of the number of adults in England, Wales, and Scotland with a hearing loss. International Journal of Audiology 2014;53(1):60-1.

\section{AoHL 2015}

Action on Hearing Loss. Hearing Matters. http:// www.actiononhearingloss.org.uk/supporting-you/policyresearch-and-influencing/research/hearing-matters.aspx (accessed 2 August 2017). London, 1-114.

\section{Barker 2016}

Barker F, Mackenzie E, Elliott L, Jones S, de Lusignan S. Interventions to improve hearing aid use in adult auditory rehabilitation. Cochrane Database of Systematic Reviews 2016, Issue 8. [DOI: 10.1002/14651858.CD010342.pub3]

\section{Barton 2004}

Barton GR, Bankart J, Davis AC, Summerfield QA. Comparing utility scores before and after hearing-aid provision. Applied Health Economics and Health Policy 2004;3(2):103-5.

\section{Bertoli 2009}

Bertoli S, Staehelin K, Zemp E, Schindler C, Bodner D, Probst R. Survey on hearing aid use and satisfaction in Switzerland and their determinants. International Journal of Audiology 2009;48:183-95.

\section{Boothroyd 2007}

Boothroyd A. Adult aural rehabilitation: what is it and does it work?. Trends in Amplification 2007;11(2):63-71.

\section{Browning 2017}

Browning GG, Howell P, Whitmer WM, Ftouh S, Chong LY, Naylor G. Unilateral versus bilateral hearing aids for bilateral hearing impairment in adults. Cochrane Database of Systematic Reviews 2017, Issue 5. [DOI: 10.1002/14651858.CD012665] 


\section{Chisolm 2005}

Chisolm TH, Abrams HB, McArdle R, Wilson RH, Boyle PJ. The WHO-DAS II: Psychometric properties in the measurement of functional health status in adults with acquired hearing loss. Trends in Amplification 2005;9(3):111-26.

\section{Chisolm 2007}

Chisolm T, Johnson CE, Danhauer JL, Portz Laural JP, Abrams HB, Lesner S, et al. A systematic review of healthrelated quality of life and hearing aids: final report of the American Academy of Audiology Task Force on the Health-Related Quality of Life Benefits of Amplification in Adults. Journal of the American Academy of Audiology 2007;18(2):151-83.

\section{CONSORT 2010}

CONSORT (Consolidated Standards of Reporting Trials). CONSORT 2010. http://www.consort-statement.org/ consort-2010.

\section{Coulson 2016}

Coulson N, Ferguson M, Henshaw H, Heffernan E. Applying theories of health behaviour and change to hearing health research: time for a new approach. International Journal of Audiology 2016;55(Suppl 3):S99-104.

\section{Covidence 2017 [Computer program]}

Veritas Health Innovation. Covidence systematic review software. Melbourne, Australia: Veritas Health Innovation, (accessed 7 February 2017).

\section{Cox 1990}

Cox RM, Gilmore C. Development of the Profile of Hearing Aid Performance (PHAP). Journal of Speech Language and Hearing Research 1990;33(2):343-57.

\section{Cox 1995}

Cox RM, Alexander GC. The abbreviated profile of hearing aid benefit. Ear and Hearing 1995;16:176-86.

\section{Cruickshanks 1998}

Cruickshanks KJ, Wiley TL, Tweed TS, Klein BEK, Klein R, MaresPerlman JA, et al. Prevalence of hearing loss in older adults in Beaver Dam, Wisconsin. The epidemiology of hearing loss study. American Journal of Epidemiology 1998;148(9):879-86.

\section{Danermark 2013}

Danermark B, Granberg S, Kramer SE, Selb M, Möller C. The creation of a comprehensive and a brief core set for hearing loss using the international classification of functioning, disability and health. American Journal of Audiology 2013;22(2):323-8.

\section{Davis 2007}

Davis A, Smith P, Ferguson M, Stephens D, Gianopoulos I. Acceptability, benefit and costs of early screening for hearing disability: a study of potential screening tests and models. Health Technology Assessment 2007;11(42):1-294.

\section{Dillon 2012}

Dillon H. Hearing Aids. 2nd Edition. New York: Thieme, 2012.

\section{Duval 2000}

Duval S, Tweedie R. Trim and fill: a simple funnel-plot-based method of testing and adjusting for publication bias in metaanalysis. Biometrics 2000;56(2):455-63.

\section{Ferguson 2016}

Ferguson MA, Brandreth M, Brassington W, Leighton $\mathrm{P}$, Wharrad $\mathrm{H}$. A randomized controlled trial to evaluate the benefits of a multimedia educational programme for first-time hearing aid users. Ear and Hearing 2016;27(2):123-36.

\section{Furlong 2001}

Furlong WJ, Feeny DH, Torrance GW, Barr RD. The Health Utilities Index (HUI) system for assessing health-related quality of life in clinical studies. Annals of Medicine 2001;33(5):375-84.

\section{Furukawa 2014}

Furukawa TA, Noma H, Caldwell DM, Honyashiki M, Shinohara K, Imai $\mathrm{H}$, et al. Waiting list may be a nocebo condition in psychotherapy trials: a contribution from network metaanalysis. Acta Psychiatrica Scandinavica 2014;130(3):181-92. [DOI: 10.1111/acps.12275]

\section{Gatehouse 1999}

Gatehouse S. A self-report outcome measure for the evaluation of hearing aid fittings and services. Health Bulletin 1999;57:424-36.

\section{Gatehouse 2004}

Gatehouse S, Noble W. The Speech, Spatial and Qualities of Hearing Scale (SSQ). International Journal of Audiology 2004;43:85-99.

\section{Granberg 2014}

Granberg S, Dahlström J, Möller C, Kähäri K, Danermark B. The ICF core sets for hearing loss-researcher perspective. Part I: Systematic review of outcome measures identified in audiological research. International Journal of Audiology 2014;53(2):65-76.

\section{Handbook 2011}

Higgins JPT, Green S (editors). Cochrane Handbook for Systematic Reviews of Interventions Version 5.1.0 [updated March 2011]. The Cochrane Collaboration, 2011. Available from www.cochrane-handbook.org.

\section{Harbour 2001}

Harbour R, Miller J. A new system for grading recommendations in evidence based guidelines. BMJ 2001;323(7308):334.

\section{Hopkins 2011}

Hopkins K, Moore BCJ. The effects of age and cochlear hearing loss on temporal fine structure sensitivity, frequency selectivity, and speech reception in noise. Journal of the Acoustical Society of America 2011;130(1):334-49.

\section{Hougaard 2011}

Hougaard S, Ruf S. Eurotrak I: A consumer survey about hearing aids in Germany, France and the UK. Hearing Review 2011;18(2):12-28. 


\section{Johnson 2016}

Johnson CE, Danhauer JL, Ellis BB, Jilla AM. Hearing aid benefit in patients with mild sensorineural hearing loss: a systematic review. Journal of the American Academy of Audiology 2016;27:293-310.

\section{Joore 2002}

Joore MA, Potjewijd J, Timmerman AA, Anteunis LJC. Response shift in the measurement of quality of life in hearing impaired adults after hearing aid fitting. Quality of Life Research 2002;11(4):299-307.

\section{Joore 2003}

Joore MA, van der Stel H, Peters HJM, Boas GM, Anteunis LJC. The cost-effectiveness of hearing-aid fitting in the Netherlands. Archives of Otolaryngology--Head \& Neck Surgery 2003;129(3):297-304.

\section{Kiessling 2003}

Kiessling J, Pichora-Fuller MK, Gatehouse S, Stephens D, Arlinger S, Chisolm T, et al. Candidature for and delivery of audiological services: special needs of older people. International Journal of Audiology 2003;42(Suppl 2):S92-101.

\section{Kirkwood 2013}

Kirkwood D. Research firm analyzes market share, retail activity, and prospects of major hearing aid manufacturers. http:// hearinghealthmatters.org/hearingnewswatch/2013/researchfirm-analyzes-market-share-retail-stores-prospects-of-majorhearing-aid-makers/ (accessed 14 December 2015) 2013.

\section{Kochkin 2009}

Kochkin S. MarkeTrak VIII: 25-year trends in the hearing health market. Hearing Review 2009;16(11):12-31.

\section{Kochkin 2010}

Kochkin S, Beck DL, Christensen LA, Compton-Conley C, Fligor BJ, Kricos PB, et al. MarkeTrak VIII: The impact of the hearing healthcare professional on hearing aid user success. Hearing Review 2010;17(4):12-34.

\section{Lin 2011}

Lin FR, Metter EJ, O'Brien RJ, Resnick SM, Zonderman AB, Ferrucci L. Hearing loss and incident dementia. Archives of Neurology 2011;68(2):214-20.

\section{Linn 1984}

Linn MW, Linn BS. Self-evaluation of life function (SELF) scale: a short, comprehensive self-report of health for elderly adults. Journal of Gerontology 1984;39:603-12.

\section{Loughrey 2015}

Loughrey D, Kelly M, Kelley G, Lawlor B, Brennan S. The association of age-related hearing loss with cognition function, cognitive impairment and dementia: a systematic review with meta-analysis. http://www.crd.york.ac.uk/PROSPERO/display_ record.asp?ID=CRD42015026052 2015.

\section{Lupsakko 2005}

Lupsakko T, Kautiainen HJ, Sulkava R. The non-use of hearing aids in people aged 75 years and over in the city of
Kuopio in Finland. European Archives of Otorhinolaryngology 2005;262:165-9.

\section{Mathers 2000}

Mathers C, Smith A, Concha M. Global burden of hearing loss in the year 2000. Global Burden of Disease 2000;18:1-30.

\section{McArdle 2006}

McArdle R, Chisolm TH, Abrams HA, Wilson RH, Boyle PJ. Erratum. The WHO-DAS II: Measuring outcomes of hearing aid intervention for adults. Trends in Amplification 2006;10(2):105.

\section{McCormack 2013}

McCormack A, Fortnum H. Why do people fitted with hearing aids not wear them?. International Journal of Audiology 2013;52(5):360-8.

\section{Mueller 2005}

Mueller GH. Fitting hearing aids to adults using prescriptive methods: an evidence-based review of effectiveness. Journal of the American Academy of Audiology 2005;16(7):448-60.

\section{Newman 1990}

Newman CW, Weinstein BE, Jacobson GP, Hug GA. The Hearing Handicap Inventory for Adults: psychometric adequacy and audiometric correlates. Ear and Hearing 1990;11:430-3.

\section{Nguyen 2017}

Nguyen M-F, Bonnefoy M, Adrait A, Gueugnon M, Petitot C, Collet $L$, et al. Efficacy of hearing aids on cognitive status of patients with Alzheimer's disease and hearing loss: a multicenter controlled randomized trial. Journal of Alzheimer's Disease 2017;58(1):123-37. [DOI: 10.3233/JAD-160793]

\section{Pichora-Fuller 2016}

Pichora-Fuller MK, Kramer SE, Eckert MA, Edwards B, Hornsby BWY, Humes LE, et al. Hearing impairment and cognitive energy: the Framework for Understanding Effortful Listening (FUEL). Ear and Hearing 2016;37:S5-S27.

\section{Picou 2013}

Picou EM, Ricketts TA, Hornsby BWY. How hearing aids, background noise, and visual cues influence objective listening effort. Ear and Hearing 2013;34(5):e52-64.

\section{Rabin 2001}

Rabin R, de Charro F. EQ-SD: a measure of health status from the EuroQol Group. Annals of Medicine 2001;33:337-43.

\section{Rabins 1999}

Rabins PV, Kasper JD, Kleinman L, Black BS, Patrick DL. Concepts and methods in the development of the ADRQL: an instrument for assessing health-related quality of life in persons with Alzheimer's disease. Journal of Mental Health 1999;5:33-48.

\section{Reese 2005}

Reese JL, Hnath-Chisolm T. Recognition of hearing aid orientation content by first-time users. American Journal of Audiology 2005;14(1):94-104. 


\section{Resnik 2009}

Resnik L, Plow MA. Measuring participation as defined by the international classification of functioning, disability and health: an evaluation of existing measures. Archives of Physical Medicine and Rehabilitation 2009;90(5):856-66.

\section{RevMan 2014 [Computer program]}

The Nordic Cochrane Centre, The Cochrane Collaboration. Review Manager (RevMan). Version 5.3. Copenhagen: The Nordic Cochrane Centre, The Cochrane Collaboration, 2014.

\section{Robinson 1996}

Robinson K, Gatehouse S, Browning GG. Measuring patient benefit from otorhinolaryngological surgery and therapy. Annals of Otology, Rhinology and Laryngology 1996;105:415-22.

\section{Solheim 2017}

Solheim J, Hickson L. Hearing aid use in the elderly as measured by datalogging and self-report. International Journal of Audiology 2017;56:466-73.

\section{Stark 2004}

Stark P, Hickson L. Outcomes of hearing aid fitting for older people with hearing impairment and their significant others. International Journal of Audiology 2004;43(7):390-8.

\section{Timmer 2015}

Timmer BHB, Hickson L, Launer S. Adults with mild hearing impairment: are we meeting the challenge?. International Journal of Audiology 2015;54(11):786-95.

\section{Tuley 1990}

Tuley MR, Mulrow CD, Aguilar C, Velez R. A critical reevaluation of the Quantified Denver Scale of Communication Function. Ear and Hearing 1990;11:56-61.

\section{Ventry 1982}

Ventry IM, Weinstein BE. The Hearing Handicap Inventory for the Elderly: a new tool. Ear and Hearing 1982;3:128-34.

\section{Ware 1992}

Ware JE, Sherbourne CD. The MOS 36-item short-form health survey (SF-36): I. Conceptual framework and item selection. Medical Care 1992;30(6):473-83.

\section{CHARACTERISTICS OF STUDIES}

Characteristics of included studies [ordered by study ID]

\section{Weinstein 1997}

Weinstein B. Outcome measures in the hearing aid fitting/ selection process. Trends in Amplification 1997;2(4):117-37.

\section{WHO 2001}

World Health Organization. International Classification of Functioning, Disability and Health (ICF). Geneva: World Health Organization, 2001.

\section{WHO 2002}

World Health Organization. Towards a common language for functioning, disability and health: The International Classification of Functioning, Disability and Health. http:// www.who.int/classifications/icf/training/icfbeginnersguide.pdf 2002.

\section{WHO 2008}

World Health Organization. The Global Burden of Disease: 2004 Update. Geneva: World Health Organization, 2008:1-146.

\section{WHO 2012a}

World Health Organization. WHO global estimates on prevalence of hearing loss. http://www.who.int/pbd/deafness/ WHO_GE_HL.pdf 2012.

\section{WHO 2012b}

World Health Organization. WHO Disability Assessment Schedule 2.0. http://www.who.int/classifications/icf/whodasii/ en/ 2012.

\section{References to other published versions of this review \\ Ferguson 2015}

Ferguson MA, Kitterick PT, Edmondson-Jones M, Hoare DJ. Hearing aids for mild to moderate hearing loss in adults. Cochrane Database of Systematic Reviews 2015, Issue 12. [DOI: 10.1002/14651858.CD012023]

* Indicates the major publication for the study

Adrait 2017

Methods 2-arm, double-blinded, multi-centre, with 6 months duration of treatment and follow-up

Participants

Location: France, multi-centre sites

Setting of recruitment and treatment: hospital setting

Sample size:

- Number randomised: $51^{\star}, 48$ fitted with hearing aids (22 intervention, 26 control) 
- Number completed: 38 (18 in intervention, 20 in comparison; attrition $n=10$ (20.8\%), caregiver withdrawal $n=1$, caregiver or legal representative withdrawal $n=2$, voluntary withdrawal $n=3$, investigator exclusion $n=1$, protocol deviation $n=2$, serious adverse event not related to the trial $n=1$ ). ${ }^{*} n=$ 3 not fitted, 2 in active group, 1 in placebo group (erroneously included (unilateral hearing loss) $n=$ 1 , voluntary withdrawal $\mathrm{n}=2$ )

\section{Participant (baseline) characteristics:}

- Age: intervention, mean 83.0 years (SD 6.2); control, mean 82.3 years (SD 7.2)

- Gender: intervention, 8 male, 14 female; control, 11 male, 15 female

- Main diagnosis: hearing loss (pure-tone average (PTA) averaged across 0.5, 1.0, 2.0, $4.0 \mathrm{kHz}$; bilateral): intervention 50.6 dB HL (SD 11.4); control mean 47.2 dB HL (SD 9.6)

- Other important effect modifiers: all patients had Alzheimer's disease (see inclusion criteria)

Inclusion criteria: probable diagnosis of AD according to DSM-IV and NINCDS-ADRDA, aged >= 65 years, Mini-Mental State Examination score between 10 and 28, bilateral SNHL (between 21 and $80 \mathrm{~dB}$ $\mathrm{HL}$ ), not worn hearing aids for previous 2 years, tolerates hearing aids for at least 1 hour per day, living with an informal, motivated caregiver.

Exclusion criteria: non-AD dementia (medical history, clinical elements, biological/medical imaging data examples given), recent introduction of cognitive-behavioural treatment, change in dosage of treatments prior to the study (cholinergic and memantine $(<6 \mathrm{~m}$ ), psychotropic medication $(<2 \mathrm{~m})$ ), recent change in dosage of treatments (cholinergic and memantine $(<2 \mathrm{~m})$, (cholinergic and memantine $(<1 \mathrm{~m}))$, break or loss of hearing aids 2 or more times during study.

Interventions

Intervention group ( $\mathbf{n}=\mathbf{1 8}$ ): active hearing aids (SAVIA and VALEO (Phonak), behind-the-ear, fully digital, bilateral fits. Fitted according to Phonak Digital (proprietary fitting algorithm derived from NAL$\mathrm{NL1}$ ).

Comparator group $(\mathbf{n}=\mathbf{2 0})$ : placebo hearing aids, programmed to minimal amplification so patients could just hear $25 \mathrm{~dB}$ SL white noise (30 dB on average), to compensate for the occlusion effect.

Use of additional interventions (common to both treatment arms): at 6 months, placebo hearing aids were activated; both groups used active hearing aids for 12 months study endpoint.

Primary outcome: Neuropsychiatric Inventory
Secondary outcomes: Instrumental Activities of Daily Living, Zarit, Alzheimer Disease Related Quality
of Life, Duke health profile (simplified, items 15 and 16 on social interactions) for patient and caregiver
Adverse effects.
None of these outcome measures were relevant or appropriate to the outcome domains of interest
specified in this review.

Funding sources French Ministry of Health (Clinical Research Hospital Program 2005, PHRC 2005-APN) and the Fondation Mederic Alzheimer, Paris

Declarations of interest Nothing to disclose

Notes All participants had a probable diagnosis of Alzheimer's disease and so this was a distinctly different
clinical population from typical first-time hearing aid users.

Hearing aids provided by Phonak at no cost to the participant.

\section{Risk of bias}

\begin{tabular}{lll}
\hline Bias & Authors' judgement & Support for judgement \\
\hline $\begin{array}{l}\text { Random sequence genera- } \\
\text { tion (selection bias) }\end{array}$ & Low risk & $\begin{array}{l}\text { Randomisation used a pre-established and well-balanced list based on } \\
\text { chronological order of inclusion. Used blocks of } 6 \text { patients. }\end{array}$ \\
\hline
\end{tabular}


Adrait 2017 (Continued)

Allocation concealment Low risk Randomisation procedure was centralised at a clinical research unit remote (selection bias) from the study setting and conducted by research methodologists independent of the study team.

$\begin{array}{ll}\begin{array}{l}\text { Blinding of participants } \\ \text { and personnel (perfor- }\end{array} & \text { Low risk } \\ \begin{array}{l}\text { mance bias) } \\ \text { All outcomes }\end{array} & \begin{array}{l}\text { Placebo hearing aids used so participants were blinded. Only the hearing aid } \\ \text { specialist fitting the devices knew the randomisation group of the participants } \\ \text { (required to apply the appropriate gain prescription). Unlikely that blinding } \\ \text { could be broken. }\end{array}\end{array}$

Outcome assessor and participants were blinded.

Blinding of outcome as-
sessment (detection bias)

All outcomes

\begin{tabular}{|c|c|c|}
\hline $\begin{array}{l}\text { Incomplete outcome data } \\
\text { (attrition bias) }\end{array}$ & Unclear risk & $\begin{array}{l}\text { Dropouts and withdrawals were documented but unclear why some data from } \\
\text { the remaining participants on some outcomes were not reported. }\end{array}$ \\
\hline
\end{tabular}

All outcomes

$\begin{array}{ll}\begin{array}{l}\text { Selective reporting (re- } \\ \text { porting bias) }\end{array} & \text { Dow risk } \\ \end{array}$

Other bias Low risk Study appears to be free of other sources of bias.

Humes 2017

Methods

Participants 3-arm, double-blinded, single-centre, parallel-arm RCT, with 6 and 10 weeks duration of treatment and follow-up

Location: USA (Indiana)

Setting of recruitment and treatment: university research clinic

\section{Sample size:}

- Number randomised: $164^{\star}$ (53 intervention (audiology best practice, $A B$ ), 55 intervention (consumer decides, CD), 55 control (placebo).

- Number completed: 154 (53 intervention (audiology best practice, AB), 50 intervention (consumer decides, $C D$ ), 51 control (placebo); attrition $n=10(6.0 \%)$, ear or health problems $n=3$, fitting problem or non-use $n=2$, unable to complete hearing aid selection $n=4$, ${ }^{*}$ withdrawal after randomisation and before the fitting session $n=1$ )

\section{Participant (baseline) characteristics:}

Age: mean 69.1 years (SD 6.1)

Gender: 92 male, 72 female

Main diagnosis: hearing loss (PTA averaged across 0.5, 1.0, $2.0 \mathrm{kHz}=28.1 \mathrm{~dB} \mathrm{HL}$ (SD 8.0); high frequency PTA averaged across 1.0, 2.0, $4.0 \mathrm{kHz}=38.8 \mathrm{~dB} \mathrm{HL}(\mathrm{SD} 7.9)$

Other important effect modifiers: none

Inclusion criteria: age 55 to 79 years, English as native language, MMSE score $>25$, no prior hearing aid experience, pure-tone audiometry (air) consistent with age-related hearing loss within the fitting guidelines of this study, bilaterally symmetrical hearing loss.

Exclusion criteria: presence of a medically treatable ear condition, bilateral, flat tympanograms, known fluctuating or progressive $\mathrm{HL}$, presence of cognitive, medical or language-based conditions that limit ability to complete all test procedures, currently or recently taking platinum-based cancer drugs or mycin-family antibiotics, previously diagnosed with either multiple sclerosis or Ménière's disease, 
Humes 2017 (Continued)

failure to seek or waive medical evaluation and clearance following hearing evaluation, unwillingness to be randomly assigned to a treatment group.

Interventions

Intervention group, $\mathbf{A B}$ and $\mathbf{C D}(\mathbf{n}=\mathbf{1 0 8})$ : active hearing aids (ReSound Alera Mini), behind-the-ear, fully digital. Bilateral fits. Fixed directional microphones, dynamic feedback suppression and noise reduction unclear if enabled. AB: fitted using real-ear measurements according to the NAL-NL2 target, with adjustments as necessary. Verified via real ear measurements using Audioscan Verifit system. CD: 3 possible prescriptions based on NAL-NL2 fit to the 3 most common patterns of hearing loss among older adults in the US. Different programmes applying different constant gains across all frequencies (gain values based on chosen typical prescription).

Comparator group ( $\mathbf{n}=\mathbf{5 1}$ ): placebo hearing aids (ReSound Alera Mini), behind-the-ear, fully digital. Bilateral fits. Fixed directional microphones $(n=20)$, omni-directional microphones $(n=23)$, dynamic feedback suppression and noise reduction enabled. Programmed to achieve $0 \mathrm{~dB}$ insertion gain to control for any occlusion effect. Verified via real ear measurements using Audioscan Verifit system.

Use of additional interventions (common to both treatment arms): none up to 6 weeks post-baseline, then the CD group was offered AB-delivered hearing aids for a further 4 to 5 weeks trial.

\begin{tabular}{ll}
\hline Outcomes & $\begin{array}{l}\text { Primary outcome: Profile of Hearing Aid Benefit } \\
\text { Secondary outcomes: Connected Speech Test, Hearing Handicap Inventory for the Elderly, Hearing } \\
\text { Aid Satisfaction Survey }\end{array}$ \\
\hline Funding sources & National Institute on Deafness and Other Communication Disorders R01 DC011771 \\
\hline Declarations of interest & None reported \\
\hline Notes & $\begin{array}{l}\text { AB and CD were combined into the intervention group as hearing aids not service delivery models were } \\
\text { of interest. The 6-week follow-up measured the primary and secondary outcome measures. }\end{array}$ \\
& Participants paid for their hearing aids. \\
\hline
\end{tabular}

\section{Risk of bias}

\begin{tabular}{|c|c|c|}
\hline Bias & Authors' judgement & Support for judgement \\
\hline $\begin{array}{l}\text { Random sequence genera- } \\
\text { tion (selection bias) }\end{array}$ & Low risk & $\begin{array}{l}\text { Based on random number generation prior to study initiation, blocked by un- } \\
\text { aided Connected Speech Test performance (low, medium, high). }\end{array}$ \\
\hline $\begin{array}{l}\text { Allocation concealment } \\
\text { (selection bias) }\end{array}$ & High risk & $\begin{array}{l}\text { The clinical trial co-ordinator (CTC) allocating patients had access to the ran- } \\
\text { domisation lists and allocation was not concealed. }\end{array}$ \\
\hline $\begin{array}{l}\text { Blinding of participants } \\
\text { and personnel (perfor- } \\
\text { mance bias) } \\
\text { All outcomes }\end{array}$ & Unclear risk & $\begin{array}{l}\text { All markings and materials revealing manufacturer or model of the devices } \\
\text { were obscured. The CTC was not blinded to patient allocation and there were } \\
\text { several opportunities where there was potential for participants to be unblind- } \\
\text { ed. }\end{array}$ \\
\hline $\begin{array}{l}\text { Blinding of outcome as- } \\
\text { sessment (detection bias) } \\
\text { All outcomes }\end{array}$ & Unclear risk & $\begin{array}{l}\text { Outcome assessor (audiologist } 4 \text { ) was blinded to allocation of intervention } \\
\text { group. However, it is unclear whether all the participants remained effectively } \\
\text { blinded. }\end{array}$ \\
\hline $\begin{array}{l}\text { Incomplete outcome data } \\
\text { (attrition bias) } \\
\text { All outcomes }\end{array}$ & Low risk & All dropouts and loss to follow-up described and reasonable. \\
\hline $\begin{array}{l}\text { Selective reporting (re- } \\
\text { porting bias) }\end{array}$ & Low risk & $\begin{array}{l}\text { Data on all outcomes in the published study protocol (NCT01788423) were re- } \\
\text { ported. The study authors provided the full data set for this review upon re- } \\
\text { quest. }\end{array}$ \\
\hline
\end{tabular}


Humes 2017 (Continued)

Other bias Low risk Study appears to be free of other sources of bias.

McArdle 2005

Methods 2-arm, non-blinded, multi-centre, parallel-group RCT, with 2 months duration of treatment and follow-up

Participants Location: USA, 4 sites (Tennessee $n=2$, Pittsburgh, Florida)

Setting of recruitment and treatment: US veterans awaiting hearing aids for the first time at Veteran Affairs Medical Centres

\section{Sample size:}

- Number randomised: 380 (189 intervention, 191 control)

- Number completed: 362 (176 in intervention, 186 in comparison; attrition $n=18$ (4.7\%), due to death $n=3$, illness $n=4$, withdrew consent $n=4$, protocol deviation $n=2$, unknown $n=5$ ). Missing data imputed.

\section{Participant (baseline) characteristics:}

- Age: mean 69.4 years (SD 9.0)

- Gender: 374 male, 6 female

- Main diagnosis: hearing loss (PTA averaged across 0.5, 1.0, 2.0, $4.0 \mathrm{kHz}=43.17 \mathrm{~dB} \mathrm{HL}$ )

- Other important effect modifiers: none

Inclusion criteria: PTA at 2.0, 3.0, $4.0 \mathrm{kHz}>=30 \mathrm{~dB} \mathrm{HL}$ in better hearing ear, Mini-Mental State Examination pass, eligible for hearing aids, no prior hearing aid experience.

Exclusion criteria: conduction or retrocochlear pathology, asymmetry (not defined), speech recognition in quiet (not defined).

Interventions

Intervention group ( $\mathbf{n}=\mathbf{1 8 9}$ ): hearing aids (manufacturer not specified), in-the-ear, analogue or fully digital, fitted 2 weeks post-baseline. Bilateral fits routine. Fitted using real-ear measurements according to the NAL-R target, with adjustments as necessary. Fitted 2 weeks post-baseline.

Comparator group ( $\mathbf{n}=\mathbf{1 9 1}$ ): waiting list controls; no hearing aids up to 10 weeks post-baseline.

Use of additional interventions (common to both treatment arms): none up to 10 weeks post-baseline, then both groups had hearing aids.

$\begin{array}{ll}\text { Outcomes } & \text { Primary outcomes: none specified } \\ \text { Secondary outcomes: none specified }\end{array}$

Reported outcomes: baseline and 2 months post-fitting: WHO-DAS II total (WHO-DAS II subscales: Communication, Participation), HHIE, APHAB Global

\begin{tabular}{ll}
\hline Funding sources & Veterans Association \\
\hline Declarations of interest & None noted \\
\hline Notes & $\begin{array}{l}10 \text { weeks after baseline, hearing aids were fitted to the control group and the study continued to } 12 \\
\text { months follow-up. }\end{array}$ \\
& Hearing aids provided at no cost.
\end{tabular}

\section{Risk of bias}


McArdle 2005 (Continued)

Bias Authors' judgement Support for judgement

Random sequence genera- Low risk tion (selection bias)

Although no information was reported in the published manuscript, inspection of the study protocol provided by the authors showed that stratified randomisation had been used. Participants were recruited in a pairs design and coin tossing decided which group the participant was allocated too (e.g. heads, Participant $A$ is in the treatment group).

Allocation concealment Unclear risk
(selection bias)

(selection bias)

\section{High risk}

Blinding of participants and personnel (performance bias)

All outcomes
No blinding, therefore patients and personnel were aware of the intervention. Possible that the hearing aid group were treated differently compared to the waiting list group in other aspects.
Blinding of outcome assessment (detection bias) All outcomes
High risk

No blinding and outcomes were likely to have been influenced by the lack of blinding.
Incomplete outcome data Low risk Detailed information provided on attrition (see Table 2) with reasons, where (attrition bias) known. Missing data were imputed using appropriate methods.
Low risk

Selective reporting (reporting bias)
No published study protocol. However, inspection of the protocol provided by the authors indicates that the published reports include all expected outcomes.

Other bias Unclear risk

Waiting list controls have a risk of overestimating the benefit of an intervention. However, all participants in the waiting lists were to receive the active intervention at the end of the randomised phase (eligibility to receive active intervention was not conditional on severity or outcomes reported during the study).

Melin 1987

Methods 2-arm, non-blinded, multi-centre, parallel-group RCT, with a 6 weeks follow-up

\section{Participants}

Location: Sweden, 1 site

Setting of recruitment and treatment: hearing clinic at a Swedish university hospital

\section{Sample size:}

- Number randomised: 39 (20 intervention, 19 control)

- Number completed: 39 (attrition 0\%)

\section{Participant (baseline) characteristics:}

- Age: mean 72.7 years (SD 10.6)

- Gender: 13 male, 26 female

- Main diagnosis: hearing loss (PTA averaged across 0.5, 1.0, 2.0, $4.0 \mathrm{kHz}$ ) left $=40.5 \mathrm{~dB} \mathrm{HL}$ (SD 11.9) right $=39.05$ (SD 13.1)

- Other important effect modifiers: none

Inclusion criteria: hearing loss to a degree that hearing aids were needed, no prior hearing aid experience, tinnitus duration for more than 6 months 
Melin 1987 (Continued)

Exclusion criteria: none noted

Interventions Intervention group $(\mathbf{n}=\mathbf{2 0})$ : hearing aids (manufacturer: Widex, Rexton, Oticon, Siemens, Philips, Danavox), unilateral fits (95\%) 6 weeks post-baseline. Fitting not specified.

Comparator group ( $\mathbf{n}=\mathbf{1 9})$ : waiting list controls, no hearing aids up to 6 weeks post-baseline

Use of additional interventions (common to both treatment arms): none up to 6 weeks post-baseline, then both groups had hearing aids

\begin{tabular}{|c|c|}
\hline Outcomes & $\begin{array}{l}\text { Primary outcomes: Hearing Scaling (easy, fairly easy, fairly difficult, difficult), } 6 \text { weeks post-fitting } \\
\text { Secondary outcomes: none specified } \\
\text { Reported outcomes: as for primary outcomes } \\
\text { Hearing Scaling assessment was not included in the analysis of listening abilities because not all of the } \\
\text { data required were available in the paper and were no longer available. }\end{array}$ \\
\hline Funding sources & $\begin{array}{l}\text { Bank of Sweden Tercentenary Foundation (grant No. 83/16) and grants from Stifrelsen, Stockholm and } \\
\text { Oticon Foundation, Copenhagen }\end{array}$ \\
\hline Declarations of interest & None noted \\
\hline Notes & $\begin{array}{l}6 \text { weeks after baseline, hearing aids were fitted to the control group and the study continued to } 10 \\
\text { weeks follow-up for each group. } \\
\text { Hearing aids were provided at no cost. }\end{array}$ \\
\hline
\end{tabular}

\section{Risk of bias}

Bias Authors' judgement Support for judgement

Random sequence genera- Low risk tion (selection bias)

Insufficient information was provided in the paper. Quote: "To prevent bias, the random allocations of the subjects were done after their first interview according to a randomisation plan".

However, contact with the authors revealed randomisation was most likely done in blocks of 10 (5 participants experimental group, 5 participants control group) to recruit groups of the same size.

$\begin{array}{ll}\begin{array}{l}\text { Allocation concealment } \\ \text { (selection bias) }\end{array} & \begin{array}{l}\text { Insufficient information was provided in the paper. Quote: "To prevent bias, } \\ \text { the random allocations of the subjects were done after their first interview ac } \\ \text { cording to a randomisation plan". }\end{array} \\ \text { However, contact with the authors revealed that the allocation to group was } \\ \text { concealed by using pre-prepared opaque envelopes. }\end{array}$

\begin{tabular}{|c|c|c|}
\hline $\begin{array}{l}\text { Blinding of participants } \\
\text { and personnel (perfor- } \\
\text { mance bias) } \\
\text { All outcomes }\end{array}$ & High risk & $\begin{array}{l}\text { No blinding, therefore patients and personnel were aware of the intervention. } \\
\text { Possible that the hearing aid group were treated differently compared to the } \\
\text { waiting list group in other aspects. }\end{array}$ \\
\hline $\begin{array}{l}\text { Blinding of outcome as- } \\
\text { sessment (detection bias) } \\
\text { All outcomes }\end{array}$ & High risk & $\begin{array}{l}\text { No blinding and outcomes were likely to have been influenced by the lack of } \\
\text { blinding. }\end{array}$ \\
\hline $\begin{array}{l}\text { Incomplete outcome data } \\
\text { (attrition bias) } \\
\text { All outcomes }\end{array}$ & Unclear risk & $\begin{array}{l}\text { No participant dropouts, but insufficient reporting of whether all data points } \\
\text { from the hearing scale were completed by group. Historic records not avail- } \\
\text { able. }\end{array}$ \\
\hline
\end{tabular}


Melin 1987 (Continued)

Selective reporting (re- Low risk Study protocol not available but the authors indicated that no measures other porting bias) than the listening scaling task were included in the study.

Other bias

Unclear risk
Waiting list controls have a risk of overestimating the benefit of an intervention.

However, all participants in the waiting lists were to receive the active intervention at the end of the experimental phase (not conditional on severity at the end of the study).

Mulrow 1990

Methods 2-arm, non-blinded, single-centre, parallel-group RCT, with 16 weeks duration of treatment and follow-up at 6 and 16 weeks

Participants Location: USA, 1 site

Setting of recruitment and treatment: US veterans undergoing hearing assessment tests at the Audie L. Murphy Memorial Veterans Hospital and associated primary care clinics

\section{Sample size:}

- Number randomised: 194 (95 intervention, 99 control)

- Number completed: 188 (92 in intervention, 96 in comparison; attrition $n=6(3.1 \%)$, due to death $n$ $=5$, moved outside the 100 mile limit $n=1$ )

\section{Participant (baseline) characteristics:}

- Age: hearing aid group $73( \pm 7)$; control group $71( \pm 5)$

- Gender: hearing aid group $100 \%$ male, $0 \%$ female; control group $99 \%$ male, $1 \%$ female

- Main diagnosis: hearing loss (hearing aid group PTA 1.0, 2.0, $4.0 \mathrm{kHz}$ better ear: $53( \pm 10) \mathrm{dB} \mathrm{HL}$; control group PTA 1.0, 2.0, $4.0 \mathrm{kHz}$ better ear: $51( \pm 8) \mathrm{dB} \mathrm{HL}$

- Other important effect modifiers: none

Inclusion criteria: PTA at $2 \mathrm{kHz}$ better ear >= $40 \mathrm{~dB}$ HL in better hearing ear, over 64 years

Exclusion criteria: severely disabling comorbid disease, current hearing aid users, live more than 100 miles from the clinic, existing hearing aid users

Interventions

Intervention group ( $\mathbf{n}=\mathbf{9 5}$ ): hearing aids (manufacturer not specified), in-the-ear $(98 \%)$, unilateral fits (97\%), typically to the worst hearing ear

Comparator group $(\mathbf{n}=99)$ : waiting list controls, no hearing aids

Use of additional interventions (common to both treatment arms): none

$\begin{array}{ll}\text { Outcomes } & \begin{array}{l}\text { Primary outcomes: none specified } \\ \text { Secondary outcomes: none specified }\end{array}\end{array}$

Reported outcomes: baseline and 16 weeks post-fitting. Hearing Handicap Inventory for the Elderly, Quantified Denver Scale, Short Portable Mental Status Questionnaire, Geriatric Depression Scale, Self Evaluation and Life Function. HHIE and QDS also measured at 6 weeks. HHIE results at 16 weeks used in meta-analyses. Physicians' Teaching and Research Scholar Award

Declarations of interest None noted


Mulrow 1990 (Continued)

Notes Hearing aids provided at no cost

\section{Risk of bias}

\begin{tabular}{lll}
\hline Bias & Authors' judgement & Support for judgement \\
\hline $\begin{array}{l}\text { Random sequence genera- } \\
\text { tion (selection bias) }\end{array}$ & Low risk & $\begin{array}{l}\text { Sequence generated using block randomisation with a block size of } 6 \text { ( } 3 \times 2 \\
\text { treatments). }\end{array}$ \\
\hline $\begin{array}{l}\text { Allocation concealment } \\
\text { (selection bias) }\end{array}$ & Low risk & $\begin{array}{l}\text { Use of blocked randomisation could have created situations in which alloca- } \\
\text { tions at the end of a block can be guessed. However, a block size of } 6 \text { ( } 3 \times 2 \\
\text { treatments) would have created sufficient uncertainty. Concealment was facil- } \\
\text { itated through use of a remote telephone allocation service. }\end{array}$
\end{tabular}

\begin{tabular}{|c|c|c|}
\hline $\begin{array}{l}\text { Blinding of participants } \\
\text { and personnel (perfor- } \\
\text { mance bias) }\end{array}$ & High risk & $\begin{array}{l}\text { No blinding, therefore patients and personnel were aware of the intervention. } \\
\text { Possible that the hearing aid group were treated differently compared to the } \\
\text { waiting list group in other aspects. }\end{array}$ \\
\hline
\end{tabular}

All outcomes

$\begin{array}{lll}\begin{array}{l}\text { Blinding of outcome as- } \\ \text { sessment (detection bias) }\end{array} & \text { High risk } & \begin{array}{l}\text { No blinding, and outcomes were likely to have been influenced by the lack of } \\ \text { blinding }\end{array}\end{array}$

All outcomes

Quote: "All scales were self-administered, except the SPMSQ which was administered by a trained interviewer".

\begin{tabular}{ll}
\hline $\begin{array}{l}\text { Incomplete outcome data } \\
\text { (attrition bias) }\end{array}$ & Low risk \\
All outcomes & $\begin{array}{l}\text { Missing outcome data due to dropouts that were even across treatment and } \\
\text { control groups and for which reasons were reported and unlikely to be related } \\
\text { to the true outcome. }\end{array}$
\end{tabular}

\begin{tabular}{ll}
\hline $\begin{array}{l}\text { Selective reporting (re- } \quad \text { Low risk } \\
\text { porting bias) }\end{array}$ & $\begin{array}{l}\text { No published study protocol. However, no evidence to support the suggestion } \\
\text { that the published reports did not include all the expected outcomes including } \\
\text { those that were pre-specified. }\end{array}$ \\
\hline
\end{tabular}

\begin{tabular}{|c|c|c|}
\hline Other bias & Unclear risk & $\begin{array}{l}\text { Waiting list controls have a risk of overestimating the benefit of an interven- } \\
\text { tion. However, all participants in the waiting list group were to receive the } \\
\text { active intervention at the end (not conditional on severity at the end of the } \\
\text { study). }\end{array}$ \\
\hline
\end{tabular}

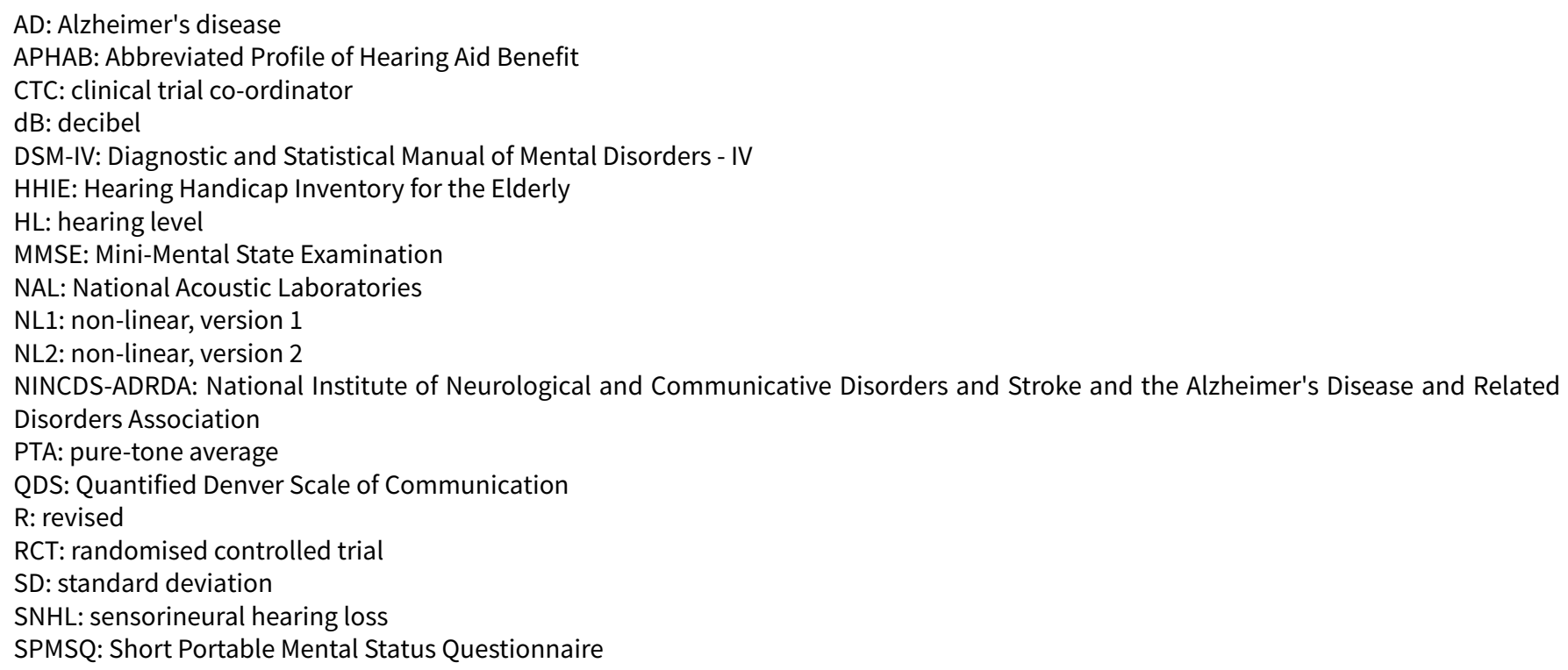


WHO-DAS: World Health Organization Disability Assessment Schedule

Characteristics of excluded studies [ordered by study ID]

\begin{tabular}{ll}
\hline Study & Reason for exclusion \\
\hline Abrams 2002 & $\begin{array}{l}\text { ALLOCATION: not true randomisation, as patients were allocated by eligibility for VA funded hear- } \\
\text { ing aids: those eligible for funding through the VA received hearing aids; those not eligible for hear- } \\
\text { ing aids acted as controls. }\end{array}$ \\
\hline Jerger 1992 & ALLOCATION: non-randomised controlled trial \\
\hline Lavie 2015 & ALLOCATION: non-randomised controlled trial \\
\hline Tolson 2002 & $\begin{array}{l}\text { ALLOCATION: randomised controlled trial } \\
\text { PARTICIPANTS: definition of hearing loss does not meet the inclusion criterion }\end{array}$ \\
\hline Yueh 2001 & $\begin{array}{l}\text { ALLOCATION: not true randomisation, as patients were allocated by eligibility for VA funded hear- } \\
\text { ing aids: those eligible for funding through the VA received hearing aids; those not eligible for hear- } \\
\text { ing aids acted as controls. }\end{array}$ \\
\hline
\end{tabular}

VA: Veterans Association

Characteristics of ongoing studies [ordered by study ID]

NCT03002142

\begin{tabular}{ll}
\hline Trial name or title & Auditory Rehabilitation and Cognition in Alzheimer Patients (RACO-MA) \\
\hline Methods & Randomised controlled trial, double-blind design \\
\hline Participants & Adults with Alzheimer's disease and hearing loss \\
\hline Interventions & Hearing aids and placebo \\
\hline Outcomes & $\begin{array}{l}\text { Alzheimer's Disease Assessment Scale - cognitive scale (primary), pure-tone and speech audiom- } \\
\text { etry, Mini-Mental State Examination, Hearing Handicap Inventory for the Elderly (HHIE), Hearing } \\
\text { Loss Impact Scale in Adults (HLSiA), Quality of life - Alzheimer's disease scale, Zarit scale for care- } \\
\text { giver burden, Geriatric Depression Scale, Trail Making Test for executive function, Wechsler Adult } \\
\text { Intelligence Scale, Beauregard test for speech comprehension, Glasgow Hearing Aid Benefit Profile } \\
\text { (GHABP). Outcomes of interest to this review are the HHIE, HLISiA and GHABP. }\end{array}$ \\
\hline Starting date & December 2016 \\
\hline Contact information & None supplied \\
\hline Notes & University Hospital, Tours, France \\
\hline
\end{tabular}

\section{DATA AND ANALYSES}


Comparison 1. Hearing aids versus no/placebo hearing aids

\begin{tabular}{|c|c|c|c|c|}
\hline Outcome or subgroup title & No. of studies & $\begin{array}{l}\text { No. of partici- } \\
\text { pants }\end{array}$ & Statistical method & Effect size \\
\hline $\begin{array}{l}1 \text { Hearing-specific health-related quality } \\
\text { of life }\end{array}$ & 3 & 722 & $\begin{array}{l}\text { Mean Difference (IV, Ran- } \\
\text { dom, } 95 \% \mathrm{CI} \text { ) }\end{array}$ & $\begin{array}{l}-26.47[-42.16 \\
-10.77]\end{array}$ \\
\hline $\begin{array}{l}\text { 1.1 Subgroup A (community setting, } \\
\text { male-female balance, behind-the-ear } \\
\text { hearing aids, placebo control) }\end{array}$ & 1 & 154 & $\begin{array}{l}\text { Mean Difference (IV, Ran- } \\
\text { dom, } 95 \% \mathrm{CI} \text { ) }\end{array}$ & $\begin{array}{l}-10.54[-15.26 \\
-5.82]\end{array}$ \\
\hline $\begin{array}{l}1.2 \text { Subgroup } B \text { (Veterans Association set- } \\
\text { ting, mostly male, in-the-ear hearing aids, } \\
\text { waiting list control) }\end{array}$ & 2 & 568 & $\begin{array}{l}\text { Mean Difference (IV, Ran- } \\
\text { dom, } 95 \% \mathrm{CI} \text { ) }\end{array}$ & $\begin{array}{l}-33.48[-36.72 \\
-30.23]\end{array}$ \\
\hline 2 Health-related quality of life & 2 & 568 & $\begin{array}{l}\text { Std. Mean Difference (IV, } \\
\text { Random, } 95 \% \mathrm{CI} \text { ) }\end{array}$ & $\begin{array}{l}-0.38[-0.55 \\
-0.21]\end{array}$ \\
\hline $\begin{array}{l}\text { 2.1 WHO Disability Assessment Schedule } \\
2.0 \text { (WHO-DAS II, range } 0 \text { to } 100 \text {, lower is } \\
\text { better) }\end{array}$ & 1 & 380 & $\begin{array}{l}\text { Std. Mean Difference (IV, } \\
\text { Random, } 95 \% \mathrm{CI} \text { ) }\end{array}$ & $\begin{array}{l}-0.44[-0.65 \\
-0.24]\end{array}$ \\
\hline $\begin{array}{l}2.2 \text { Self-evaluation of Life Function (SELF, } \\
\text { range } 54 \text { to } 216 \text {, lower is better) }\end{array}$ & 1 & 188 & $\begin{array}{l}\text { Std. Mean Difference (IV, } \\
\text { Random, } 95 \% \mathrm{CI} \text { ) }\end{array}$ & $-0.26[-0.55,0.03]$ \\
\hline 3 Listening ability & 2 & 534 & $\begin{array}{l}\text { Std. Mean Difference (IV, } \\
\text { Random, } 95 \% \mathrm{CI} \text { ) }\end{array}$ & $\begin{array}{l}-1.88[-3.24 \\
-0.52]\end{array}$ \\
\hline $\begin{array}{l}\text { 3.1 Profile of Hearing Aid Performance } \\
\text { (PHAP, range } 0 \text { to } 1 \text {, lower is better) }\end{array}$ & 1 & 154 & $\begin{array}{l}\text { Std. Mean Difference (IV, } \\
\text { Random, } 95 \% \mathrm{CI} \text { ) }\end{array}$ & $\begin{array}{l}-1.18[-1.54 \\
-0.81]\end{array}$ \\
\hline $\begin{array}{l}\text { 3.2 Abbreviated Profile of Hearing Aid } \\
\text { Benefit (APHAB, range } 0 \text { to } 100 \text {, lower is } \\
\text { better) }\end{array}$ & 1 & 380 & $\begin{array}{l}\text { Std. Mean Difference (IV, } \\
\text { Random, } 95 \% \mathrm{CI} \text { ) }\end{array}$ & $\begin{array}{l}-2.57[-2.84 \\
-2.30]\end{array}$ \\
\hline
\end{tabular}

\section{Analysis 1.1. Comparison 1 Hearing aids versus no/placebo hearing aids, Outcome 1 Hearing-specific health-related quality of life.}

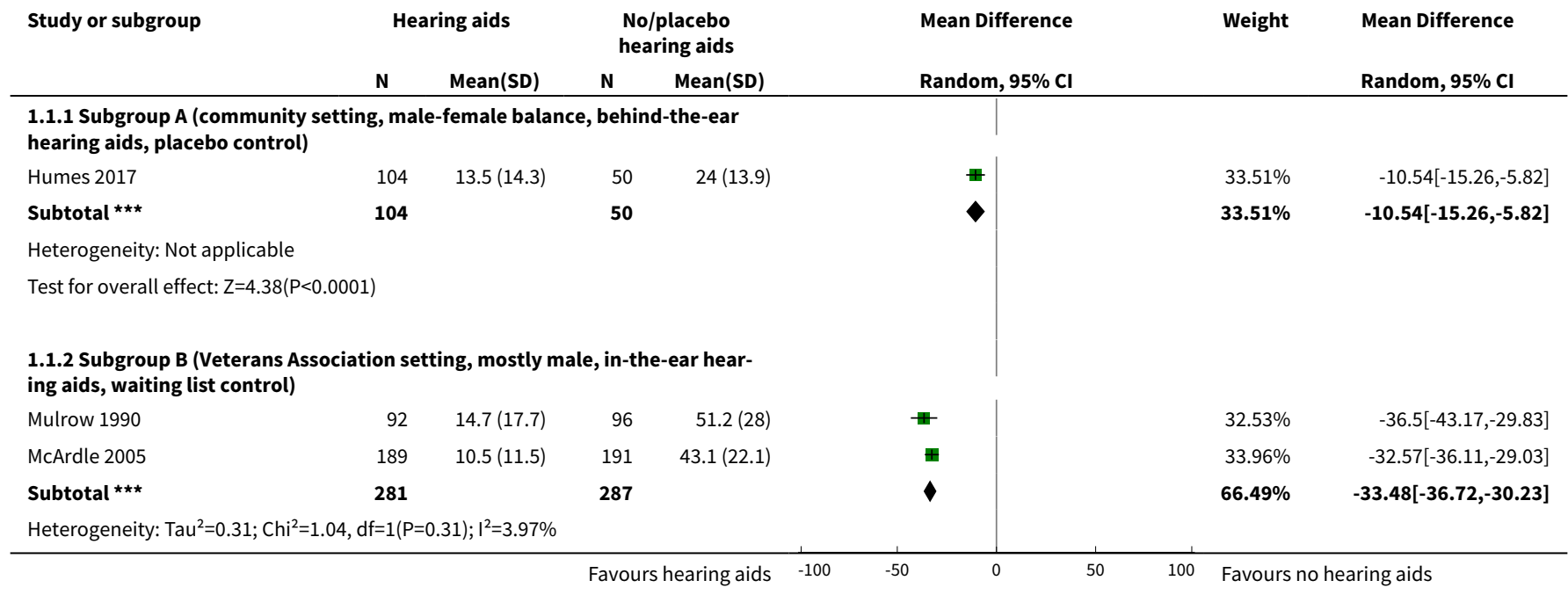




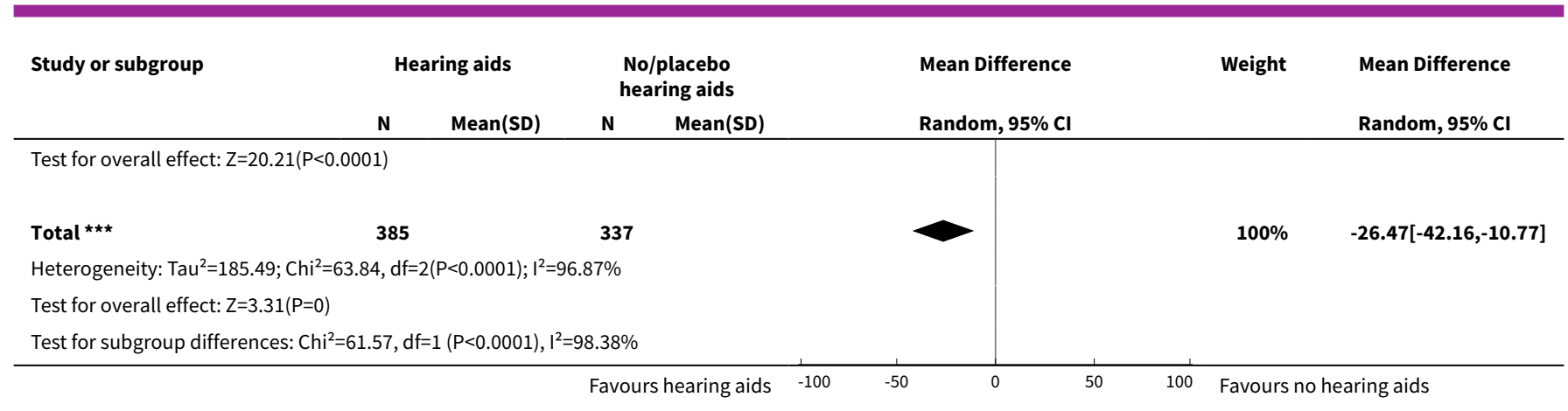

Analysis 1.2. Comparison 1 Hearing aids versus no/placebo hearing aids, Outcome 2 Health-related quality of life.

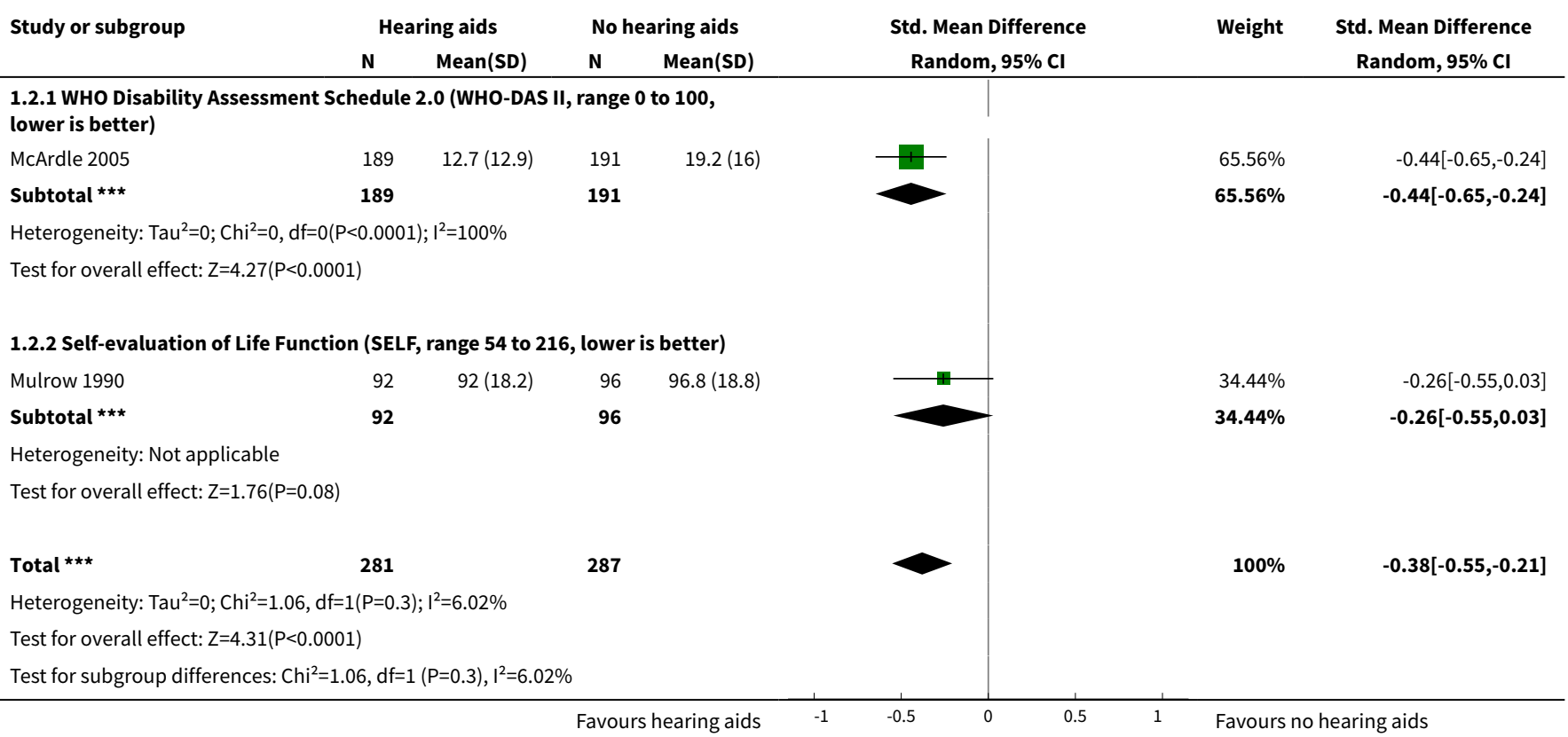

Analysis 1.3. Comparison 1 Hearing aids versus no/placebo hearing aids, Outcome 3 Listening ability.

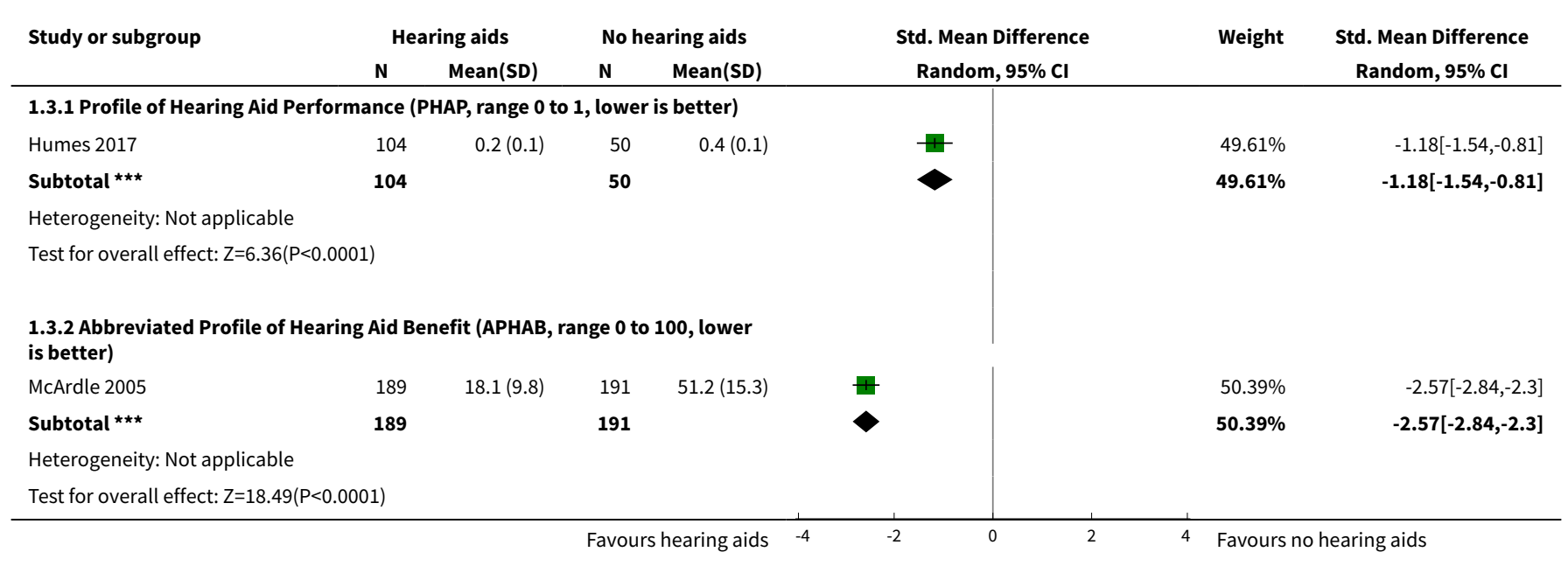




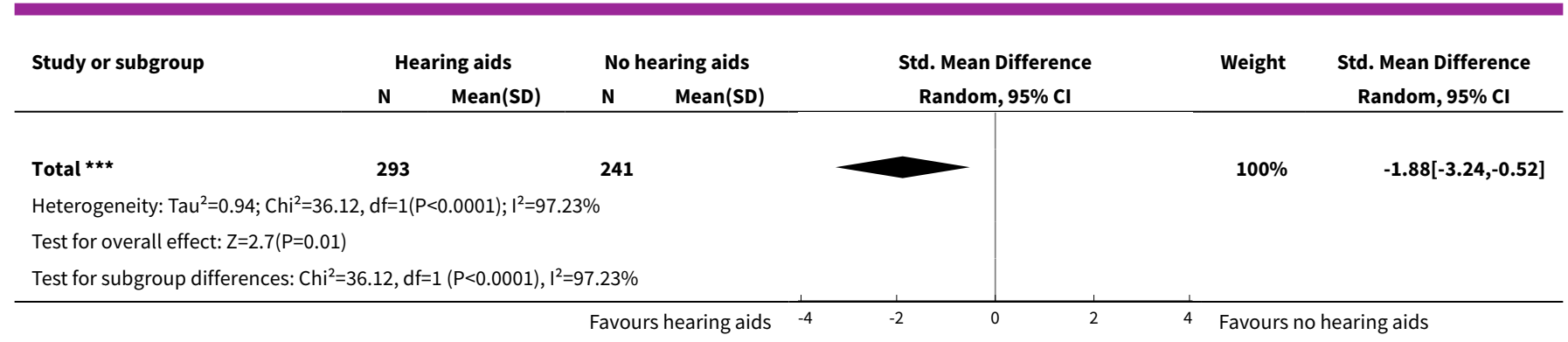

\section{APPENDICES}

\section{Appendix 1. Search strategies}

\begin{tabular}{|c|c|c|}
\hline CRSO & MEDLINE (Ovid) & Embase (Ovid) \\
\hline $\begin{array}{l}\text { \#1 MESH DESCRIPTOR Hearing Loss EXPLODE } \\
\text { ALLTREES }\end{array}$ & 1 exp Hearing Loss/ & 1 exp hearing impairment/ \\
\hline $\begin{array}{l}\text { \#2 MESH DESCRIPTOR Persons With Hearing } \\
\text { Impairments EXPLODE ALL TREES }\end{array}$ & $\begin{array}{l}2 \text { exp Persons With Hearing Impair- } \\
\text { ments/ }\end{array}$ & $\begin{array}{l}2 \text { (hearing adj3 (loss or impair*)).ti,ab. } \\
3 \text { "deaf*".ti,ab. }\end{array}$ \\
\hline \#3 (hearing near (loss or impair $\left.{ }^{\star}\right)$ ):TI,AB,KY & $\begin{array}{l}3(\text { hearing adj3 (loss or im- } \\
\left.\text { pair }^{\star}\right) \text { ).ab,ti. }\end{array}$ & 41 or 2 or 3 \\
\hline \#4 deaf*:TI,AB,KY & 4 "deaf*".ab,ti. & 5 hearing aid/ \\
\hline \#5 \#1 OR \#2 OR \#3 OR \#4 & 51 or 2 or 3 or 4 & $\begin{array}{l}6 \text { ("hearing aid }{ }^{\star "} \text { or hearing-aid* or "hear- } \\
\text { ing device*" or "hearing instrument*" or }\end{array}$ \\
\hline \#6 MESH DESCRIPTOR Hearing Aids & 6 Hearing Aids/ & "hearing system*").ti,ab. \\
\hline $\begin{array}{l}\text { \#7 ("hearing aid*" or hearing-aid* or "hearing } \\
\text { device*" or "hearing instrument*" or "hearing } \\
\text { system*"):TI,AB,KY }\end{array}$ & $\begin{array}{l}7 \text { (hearing adj3 (loss or impair }{ }^{\star} \text { ) } \\
\text { adj6 (amplif* or aided or unaided or } \\
\text { aid)).ab,ti. }\end{array}$ & $\begin{array}{l}7 \text { (hearing adj3 (loss or impair*) adj6 (am- } \\
\text { plif* or aided or unaided or aid)).ti,ab. } \\
85 \text { or } 6 \text { or } 7\end{array}$ \\
\hline $\begin{array}{l}\text { \#8 (hearing near (loss or impair }{ }^{\star} \text { ) near (amplif* } \\
\text { or aided or unaided or aid)):TI,AB,KY }\end{array}$ & $\begin{array}{l}8 \text { ("hearing aid*" or hearing-aid* or } \\
\text { "hearing device*" or "hearing instru- } \\
\text { ment }^{\star " ~ o r ~ " h e a r i n g ~ s y s t e m * ") . a b, t i . ~}\end{array}$ & 94 and 8 \\
\hline \multirow{2}{*}{ \#10 \#5 AND \#9 } & 96 or 7 or 8 & \\
\hline & 105 and 9 & \\
\hline CINAHL & LILACS & Trial Registries \\
\hline S9 S4 AND S8 & $\begin{array}{l}\text { ((((TW:hearing OR TW:Auditiva) } \\
\text { AND (TW:loss OR TW:impair* OR }\end{array}$ & ClinicalTrials.gov \\
\hline $\begin{array}{l}\text { S8 S5 OR S6 OR S7 } \\
\text { S7 TX (hearing n3 (loss or impair }{ }^{\star} \text { n6 (amplif* } \\
\text { or aided or unaided)) }\end{array}$ & 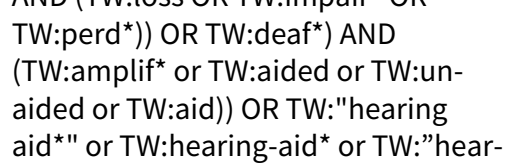 & $\begin{array}{l}\text { "hearing aid" OR "hearing aids" OR } \\
\text { (("hearing loss" OR "hearing impair- } \\
\text { ment") AND (amplification OR aided OR } \\
\text { aid OR unaided)) }\end{array}$ \\
\hline $\begin{array}{l}\text { S6 TX "hearing aid*" or hearing-aid* or "hear- } \\
\text { ing device*" or "hearing instrument*" or "hear- }\end{array}$ & $\begin{array}{l}\text { ing device*" or TW:"hearing instru- } \\
\text { ment*" or TW:"hearing system }^{\star} \text { OR }\end{array}$ & OR \\
\hline ing system*" & $\begin{array}{l}\text { TW:Audífonos OR TW:"Auxiliares de } \\
\text { Audição" }\end{array}$ & $\begin{array}{l}\text { Intervention: "hearing aid" OR "hearing } \\
\text { aids" OR (("hearing loss" OR "hearing im- }\end{array}$ \\
\hline S5 (MH "Hearing Aids") & & $\begin{array}{l}\text { pairment") AND (amplification OR aided } \\
\text { OR aid OR unaided)) }\end{array}$ \\
\hline S4 S1 OR S2 OR S3 & & \\
\hline
\end{tabular}


(Continued)

S3 TX deaf*

S2 TX hearing n3 (loss or impair ${ }^{\star}$ )

S1 (MH "Hearing Disorders") OR (MH "Deaf-

ness") OR (MH "Hearing Loss, Partial+")

\section{ICTRP}

hearing aid* or hearing-aid* or "hearing device*" or "hearing instrument" "hearing system*"

\section{CONTRIBUTIONS OF AUTHORS}

MF conceived the review question, selected which studies to review, extracted data, assessed study quality, wrote the protocol and review, and co-ordinated comments from the authors and reviewers.

PK extracted data, assessed study quality, conducted the data analyses, and contributed substantially to the writing of the protocol and review.

LY provided methodological advice on the protocol and the review.

MEJ provided statistical advice and conducted an independent verification of the data analyses.

FB joined as a co-author for the full review, selected which studies to include and provided feedback on the review.

DH selected which studies to include, assessed study quality, and provided guidance and critical comment on the draft protocol and review.

\section{DECLARATIONS OF INTEREST}

Melanie Ferguson: none known.

Pádraig T Kitterick: PK's employing organisation has received financial support from Cochlear Europe Ltd. and support in kind from Phonak UK Ltd. to conduct a multicentre clinical trial of cochlear implantation in single-sided deafness co-ordinated by PK. PK has accepted the hospitality of Cochlear Europe Ltd. to attend and speak at national and international scientific meetings.

Lee Yee Chong: none known.

Mark Edmondson-Jones: none known.

Fiona Barker: none known

Derek Hoare: none known.

\section{SOURCES OF SUPPORT}

\section{Internal sources}

- National Institute for Health Research, UK.

MF, PK, MEJ and DH are funded by the National Institute for Health Research (NIHR) Biomedical Research Unit Programme, however the views expressed are those of the authors and not necessarily those of the NHS, the NIHR or the Department of Health

\section{External sources}

- National Institute for Health Research, UK.

Infrastructure funding for Cochrane ENT

\section{DIFFERENCES BETWEEN PROTOCOL AND REVIEW}

The Types of participants section in the protocol was: "Adults ( $\geq 18$ years old) with mild or moderate hearing loss, as defined by pure-tone thresholds in the better-hearing ear averaged across four frequencies $(0.5 \mathrm{kHz}, 1.0 \mathrm{kHz}, 2.0 \mathrm{kHz}$ and $4.0 \mathrm{kHz})$ of 26 to $40 \mathrm{~dB} \mathrm{HL}$ (mild hearing loss) and 41 to $60 \mathrm{~dB} \mathrm{HL}$ (moderate hearing loss). In the absence of confirmation that all participants in a study met these criteria (i.e. where participant-level data were not reported or could not be obtained), we will include studies where the reported participant characteristics for the mean four-frequency average as described above. If a mean frequency average is offered for a combination of frequencies other than $0.5 \mathrm{kHz}, 1.0 \mathrm{kHz}, 2.0 \mathrm{kHz}$ and $4.0 \mathrm{kHz}$, we will use studies where the reported value falls between 26 to $40 \mathrm{~dB} \mathrm{HL}$ (mild hearing loss) or 41 to $60 \mathrm{~dB} \mathrm{HL}$ (moderate hearing loss). If only qualitative descriptions of mild and moderate hearing loss are given with no supporting

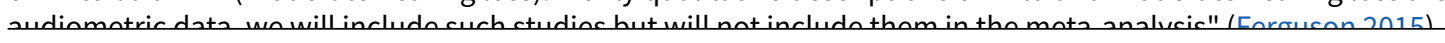


We revised the Types of participants section because there was concern about introducing a potential bias in the selection of studies such that those studies that were reported in less detail (e.g. group mean hearing threshold data or qualitative descriptions) were more likely to be included than those that either reported in more detail or provided us with participant-level data. To minimise the risk of bias from the revision of the protocol, the revised definition for Types of participants and the final decisions about which studies met the inclusion criteria were subjected to an independent review by an external expert committee to ensure the decisions were appropriate for the review question.

The protocol stated that MF and FB would extract the data, but instead this was completed by MF and PK. The protocol did not state what would happen in the event of a disagreement on a risk of bias judgement between MF and PK. Where this occurred, $\mathrm{DH}$ was charged with making a final judgement. The protocol stated that MF would review the 'Summary of findings' table, but in addition LYC (who was not an author on the review protocol) also reviewed the 'Summary of findings' table.

The protocol stated in Measures of treatment effect that we would use the standardised mean difference at the trial endpoint. We instead used the mean difference for the hearing-specific health-related quality of life outcome at the trial endpoint as these data were collected using the same instrument (HHIE) across all studies that were included in the meta-analysis.

Some of the pre-specified subgroup and funnel plot analyses were not performed as the data were not available or were too limited.

We performed unplanned subgroup analyses for the hearing-specific quality of life and listening ability outcomes due to the numerous participant and methodological differences between the Veterans Association studies and the other study included in the analyses (Humes 2017).

We made a minor adjustment to the wording of the review Objectives, changing 'effectiveness' to 'effects' to capture our interest in both positive and negative outcomes.

\section{N D EX TERMS}

\section{Medical Subject Headings (MeSH)}

${ }^{\star}$ Hearing Aids [adverse effects]; *Quality of Life; Activities of Daily Living; Hearing Loss [ ${ }^{\star}$ rehabilitation]; Randomized Controlled Trials as Topic

\section{MeSH check words}

Aged; Aged, 80 and over; Humans 